\title{
Symmetry protected gapless $Z_{2}$ spin liquids
}

\author{
Yuan-Ming $\mathrm{Lu}^{1}$ \\ ${ }^{1}$ Department of Physics, The Ohio State University, Columbus, OH 43210, USA
}

(Dated: January 1, 2018)

\begin{abstract}
Despite rapid progress in understanding gapped topological states, much less is known about gapless topological phases of matter, especially in strongly correlated electrons. In this work we discuss a large class of robust gapless quantum spin liquids in frustrated magnets made of halfinteger spins, which are described by gapless fermionic spinons coupled to dynamical $Z_{2}$ gauge fields. Requiring $U(1)$ spin conservation, time reversal and certain space group symmetries, we show that certain spinon symmetry fractionalization class necessarily leads to a gapless spectrum. These gapless excitations are stable against any perturbations, as long as the required symmetries are preserved. Applying these gapless criteria to spin one-half systems on square, triangular and kagome lattices, we show that all gapped symmetric $Z_{2}$ spin liquids in Abrikosov-fermion representation can also be realized in Schwinger-boson representation. This leads to 64 gapped $Z_{2}$ spin liquids on square lattice, and 8 gapped states on both kagome and triangular lattices.
\end{abstract}

PACS numbers:

\section{Contents}

\section{Introduction}

II. Parton construction for symmetric $Z_{2}$ spin liquids of half-integer spins

A. A general parton construction for half-integer spins

B. A brief review on symmetry fractionalization in $Z_{2}$ spin liquids

C. Spinon pair superfluid description of $Z_{2}$ spin liquids

III. Criteria for symmetry protected gapless $Z_{2}$ spin liquids

A. Two perpendicular mirror planes intersecting at one half-integer spin

B. Mirror plane crossing an odd number of half-integer spins

C. $C_{2 n}$ rotation or inversion centered at a half-integer spin

IV. Gapped and gapless $Z_{2}$ spin liquids on the square lattice

V. Gapped and gapless $Z_{2}$ spin liquids on the triangular lattice

VI. Gapped and gapless $Z_{2}$ spin liquids on the kagome lattice

\section{Discussions}

\section{Acknowledgments}

References

\section{INTRODUCTION}

Tremendous progress has been made in understanding the interplay of symmetry and topology in gapped quantum many-body phases ${ }^{1}[3$ since the discovery of topological insulators 4 6. Take two spatial dimensions for example, a complete classification has been achieved ${ }^{7 \cdot 16}$ for a generic gapped phase of interacting bosons that preserves certain (global) symmetries, characterized by possible fractional statistics and quantum numbers carried by elementary bulk and edge excitations therein. On the other hand, much less is known about gapless quantum phases beyond the free fermions regime ${ }^{17 / 18}$.

In this work we discuss a class of gapless and highly entangled quantum paramagnets in half-integer-spin systems, namely gapless $Z_{2}$ spin liquids $\frac{19 \mid 20}{}$, whose lowenergy physics is captured by an emergent $Z_{2}$ gauge field $\frac{2122}{122}$ coupled to a pair superfluid (or superconductor) of fermionic "spinons". Unlike integer-spin magnon excitations, spinons carry a half-integer spin each, and form Kramers doublets as a projective representation of the time reversal symmetry. Therefore spinons are "fractionalized" excitations, each of which can be viewed as half of a magnon. Pairings between spinons can usually destroy the spinon fermi surface, leading to a $Z_{2}$ spin liquid with a gapped spectrum $\frac{19}{19}$. Here we show that with time reversal and $U(1)$ spin rotational symmetries, certain space group symmetry implementations on spinons can give rise to a nodal superconductor of spinons with a gapless spectrum. These gapless $Z_{2}$ spin liquids are stable against any perturbations including arbitrary interactions between spinons, as long as the associated symmetries are preserved.

Between these spinon superconductors $\left(Z_{2}\right.$ spin liquids) and usual electronic superconductors, there is a crucial difference on how space group symmetries can be implemented on spinons and on electrons. Since spinons are 
"fractionalized" collective excitations of the spin system, they can carry a fractional quantum number of spatial (and global) symmetries, a phenomena known as "symmetry fractionalization' 10 . The possible symmetry fractionalization classes of bosonic (or fermionic) spinons in a $Z_{2}$ spin liquid are classified by 2 nd group cohomology $\mathcal{H}^{2}\left(G_{s}, Z_{2}\right)$ of the symmetry group ${ }^{10114} G_{s}$. In this work, we show that certain fractionalization classes necessarily lead to gapless $Z_{2}$ spin liquids on a given lattice.

Previous studies on nodal superconductors ${ }^{23124}$ and gapless $Z_{2}$ spin liquids 20 are based on perturbative arguments at the free fermion level, i.e. by analyzing symmetry-allowed mass terms for certain Dirac-like spectra. A natural step further is to address this question in a generic interacting system: can interactions between fermions (spinons) destroy a nodal superconductor (gapless $Z_{2}$ spin liquid) in a non-perturbative fashion? In this work, by utilizing $U(1)$ spin rotational symmetry, we are able to establish the stability of gapless spinon spectrum non-perturbatively, based on properties of Schmidt decomposition ${ }^{25}$ of a symmetric short-range-entangled ground state.

After establishing three symmetry criteria for gapless $Z_{2}$ spin liquids, we apply them to identify gapless $Z_{2}$ spin liquid $\mathrm{s}$ on square, triangular and kagome lattices. They also enable us to identify the gapped symmetric $Z_{2}$ spin liquids on these lattices, where the kagome lattice case is experimentally relevant to the possibly gapped paramagnetic ground state of Herbertsmithite $e^{2829}$. We found that on all three lattices, the only possible gapped symmetric $Z_{2}$ spin liquids are those realized in Schwingerboson representation 30 32.

This work is organized as follows. In section II we setup a generalized parton construction (1) for a half-integer spin system, using which the $Z_{2}$ spin liquids will be constructed and investigated in this paper. This representation reduces to the Schwinger-boson ${ }^{33}$ and Abrikosovfermion representation ${ }^{34}$ in the spin- $\frac{1}{2}$ case. In section III we establish three symmetry criteria (38), $(58)$ and (71)-(72) for gapless $Z_{2}$ spin liquids, by supplementing intuitive single-particle proofs with non-perturbative arguments with respect to interactions. Next we apply these criteria to symmetric $Z_{2}$ spin liquids on square (section IV), triangular (section V) and kagome (section VI) lattices, to identify the gapped and gapless $Z_{2}$ spin liquids on these lattices. Finally we conclude in section VII

\section{PARTON CONSTRUCTION FOR SYMMETRIC $Z_{2}$ SPIN LIQUIDS OF HALF-INTEGER SPINS}

\section{A. A general parton construction for half-integer spins}

In this section we setup a general parton construction for half-integer spins, which allows us to construct an arbitrary ground state in a half-integer-spin system. In particular in the case of spin- $\frac{1}{2}$ system, it becomes the familiar Schwinger-boson ${ }^{33}$ or Abrikosov-fermion ${ }^{34}$ representation. In fact this parton construction also applies to integer spins, such as spin-1 systems 35 . However in this work, we will focus on half-integer spins which give rise to gapless $Z_{2}$ spin liquids. Hereafter we will always assume $2 S=1 \bmod 2$ unless specified otherwise.

In this construction, each spin- $S(2 S \in \mathbb{Z})$ operator $\overrightarrow{\mathbf{S}}_{\mathbf{r}}$ is represented by $2 S$ pairs of spin- $\frac{1}{2}$ fermionic (or bosonic) "partons" $\left\{\phi_{\mathbf{r}, a, \alpha} \mid 1 \leq a \leq 2 S, \alpha=\uparrow, \downarrow\right\}$, where $\mathbf{r}$ label a specific lattice site:

$$
\overrightarrow{\mathbf{S}}_{\mathbf{r}}=\frac{1}{2} \sum_{a=1}^{2 S} \sum_{\alpha, \beta=\uparrow, \downarrow} \phi_{\mathbf{r}, a, \alpha}^{\dagger} \overrightarrow{\boldsymbol{\sigma}}_{\alpha, \beta} \phi_{\mathbf{r}, a, \beta}, \quad 2 S \in \mathbb{Z} .
$$

and $\overrightarrow{\boldsymbol{\sigma}}$ represent the three Pauli matrices. $a$ is the flavor index and $\alpha, \beta$ are spin indices. These partons physically correspond to spinon excitations in a quantum spin liquid, each of which carries spin- $1 / 2$ and can be viewed as half of a magnon.

Following the rule for addition of angular momentum, to ensure that $\overrightarrow{\mathbf{S}}_{\mathbf{r}}$ represents a half-integer spin we require that $2 S$ to be an odd integer and

$$
\sum_{a, \alpha} \phi_{\mathbf{r}, a, \alpha}^{\dagger} \phi_{\mathbf{r}, a, \alpha}=2 S, \quad \forall \mathbf{r} .
$$

Note that we have not specify the statistics of spinons $\left\{\phi_{\mathbf{r}, a, \alpha}\right\}$. Bose versus Fermi statistics of partons will lead to two different parton constructions for the same spin$S$ system, such as Schwinger-boson versus Abrikosovfermion representation for the $S=\frac{1}{2}$ case.

Once the parton Hamiltonian $\hat{H}_{\text {parton }}$ for $\left\{\phi_{\mathbf{r}, a, \alpha}\right\}$ is constructed and its ground state $\left|\Phi_{\text {parton }}\right\rangle$ obtained, the many-spin state $\left|\Psi_{\text {spin }}\right\rangle$ is achieved by implementing certain projection operators $\left(\hat{P}_{\mathbf{r}}\right.$ on site $\left.\mathbf{r}\right)$ on the parton ground state

$$
\left|\Psi_{\text {spin }}\right\rangle=\left(\prod_{\mathbf{r}} \hat{P}_{\mathbf{r}}\right)\left|\Phi_{\text {parton }}\right\rangle
$$

This is because the Hilbert space of partons $\left\{\phi_{\mathbf{r}, a, \alpha} \mid 1 \leq\right.$ $a \leq 2 S, \alpha=\uparrow, \downarrow\}$ per site is larger than the $(2 S+1)$ dimensional spin- $S$ Hilbert space, and hence we need to impose certain constraints on the parton Hilbert space to reduce it to the physical spin- $S$ Hilbert space. For example, condition (2) is one necessary constraint for a general parton construction (1) of spin- $S$ systems. All the constraints are enforced by a projection operator $\left\{\hat{P}_{\mathbf{r}}\right\}$ in (3) to produce a physical spin- $S$ wavefunction.

In the $S=1 / 2$ case, the generalized parton construction (1) reduces to the widely-adopted Abrikosovfermion ${ }^{34 / 38}$ or Schwinger-boson ${ }^{33}$ representation, depending on whether partons $\left\{\phi_{\mathbf{r}, a, \alpha}\right\}$ obey Fermi or Bose statistics. The on-site constraint for $S=1 / 2$ case becomes the single-occupancy condition

$$
S=\frac{1}{2} \Longrightarrow \sum_{\alpha} \phi_{\mathbf{r}, \alpha}^{\dagger} \phi_{\mathbf{r}, \alpha}=1, \quad \forall \mathbf{r} .
$$


and the associated projection operator is nothing but the well-known Gutzwiller projector

$$
\prod_{\mathbf{r}} \hat{P}_{\mathbf{r}}\left(S=\frac{1}{2}\right)=\prod_{\mathbf{r}}\left(1-\sum_{\alpha} \phi_{\mathbf{r}, \alpha}^{\dagger} \phi_{\mathbf{r}, \alpha}\right) \equiv \hat{P}_{G}
$$

In the $2 S>1$ case, the onsite constraints are typically more complicated $\sqrt{35 \sqrt[37]{37}}$ than condition (2). However for the purpose of this work, it turns out that constraint (2) itself suffices to establish the stability for gapless $Z_{2}$ spin liquids of half-integer spins. Therefore we will not bother to explicitly write down the general constraints for the parton construction (1) of an arbitrary spin- $S$ system.

\section{B. A brief review on symmetry fractionalization in $Z_{2}$ spin liquids}

Now that the physical many-spin wavefunction $\left|\Psi_{\text {spin }}\right\rangle$ is related to the many-body state $\left|\Phi_{\text {parton }}\right\rangle$ of partons $\left\{\phi_{\mathbf{r}, a, \alpha}\right\}$ by the projection (3), how different are these two wavefunctions? Do they share the same symmetry properties? Take $Z_{2}$ spin liquids for example, in order to obtain a spin wavefunction $\left|\Psi_{\text {spin }}\right\rangle$ for a $Z_{2}$ spin liquid that preserves a symmetry group $G_{s}$, what are the symmetries required for the corresponding parton Hamiltonian $\hat{H}_{\text {parton }}$ and associated parton state $\left|\Phi_{\text {parton }}\right\rangle$ ?

In the general parton construction (1), it is easy to notice that both the physical spin operators (1) and the onsite constraints (2) are invariant under certain gauge transformations on partons $\left\{\phi_{\mathbf{r}, a, \alpha}\right\}$. Two wellknown examples are the $S U(2)$ gauge transformations ${ }^{38}$ $\left\{W_{\mathbf{r}} \in S U(2)\right\}$ for Abrikosov-fermion representation of spin- $1 / 2$ :

$$
\begin{gathered}
\left(\begin{array}{c}
\phi_{\mathbf{r}, \uparrow} \\
\phi_{\mathbf{r}, \downarrow}^{\dagger}
\end{array}\right) \longrightarrow W_{\mathbf{r}}\left(\begin{array}{c}
\phi_{\mathbf{r}, \uparrow} \\
\phi_{\mathbf{r}, \downarrow}^{\dagger}
\end{array}\right), \quad W_{\mathbf{r}}=e^{\mathrm{i} \theta_{\mathbf{r}} \hat{\mathbf{n}}_{\mathbf{r}} \cdot \vec{\sigma}} \in S U(2), \\
S=1 / 2, \quad\left\{\phi_{\mathbf{r}, \alpha}\right\} \text { are Abrikosov fermions. }
\end{gathered}
$$

and the $U(1)$ gauge transformations $30131\left\{e^{\mathrm{i} \theta_{\mathbf{r}}} \in U(1)\right\}$ for Schwinger-boson representation of spin- $1 / 2$ :

$$
\begin{gathered}
\phi_{\mathbf{r}, \alpha} \longrightarrow e^{\mathrm{i} \theta_{\mathbf{r}}} \phi_{\mathbf{r}, \alpha}, \quad e^{\mathrm{i} \theta_{\mathbf{r}}} \in U(1), \\
S=1 / 2, \quad\left\{\phi_{\mathbf{r}, \alpha}\right\} \text { are Schwinger bosons. }
\end{gathered}
$$

For a given parton construction, these gauge transformations are featured by a gauge group $G_{g}^{(0)}$, which e.g. is $S U(2)$ for spin-1/2 Abrikosov-fermion case and $U(1)$ for spin-1/2 Schwinger-boson case.

As a consequence of these gauge redundancies, the parton state $\left|\Phi_{\text {parton }}\right\rangle$ may not faithfully preserve the symmetry group $G_{s}$ of the physical spin wavefunction $\left|\Psi_{\text {spin }}\right\rangle$. Most generally, the symmetry implementations on partons form a projective representation ${ }^{10[19}$ of the symmetry group $G_{s}$. In the case of a $Z_{2}$ spin liquid, the original gauge group $G_{g}^{(0)}$ is broken down to a subgroup (labeled "invariant gauge group" or IGG ${ }^{19}$ ) $\mathrm{IGG}=Z_{2}$.
And the different projective representations of the symmetry group $G_{s}$ is mathematically classified by the 2nd group cohomology $\mathcal{H}^{2}\left(G_{s}, \mathrm{IGG}=Z_{2}\right)$.

More concretely, we demonstrate the projective implementation of a physical symmetry $g \in G_{s}$ in the general parton construction (1). The symmetry action $U_{g}$ on partons $\left\{\phi_{\mathbf{r}, a, \alpha}\right\}$ is generally a combination of physical symmetry $g$ and its associated gauge rotation $\left\{W_{g}(\mathbf{r}) \in G_{g}\right\}$ :

$$
\begin{gathered}
\hat{U}_{g} \equiv \prod_{\mathbf{r}} W_{g}(\mathbf{r}) \cdot g, \\
\hat{U}_{g} \phi_{\mathbf{r}, a, \alpha} \hat{U}_{g}^{-1}=W_{g}(\hat{g} \mathbf{r}) \phi_{\hat{g} \mathbf{r}, a, \hat{g} \alpha} W_{g}^{-1}(\hat{g} \mathbf{r}) .
\end{gathered}
$$

While physical symmetries $g, h$ and their product $g h \equiv$ $g \cdot h$, their associated implementations on partons satisfy the following condition

$$
\hat{U}_{g} \cdot \hat{U}_{h}=\hat{\Omega}(g, h) \cdot \hat{U}_{g h}, \quad \hat{\Omega}(g, h) \in \mathrm{IGG} .
$$

The symmetry implementations $\left\{\hat{U}_{g} \mid g \in G_{s}\right\}$ on partons $\left\{\phi_{\mathbf{r}, a, \alpha}\right\}$ form a so-called "projective symmetry group" or PSG 19 , which is an extension of symmetry group $G_{s}$ satisfying

$$
\mathrm{PSG} / G_{s}=\mathrm{IGG} .
$$

In the case of $Z_{2}$ spin liquids, the Invariant Gauge Group takes a particularly simple form $\left(\hat{N}_{\phi}\right.$ is the total number of partons/spinons)

$$
\mathrm{IGG}=\left\{(-1)^{\hat{N}_{\phi}}, \hat{1}\right\} \simeq Z_{2}, \quad \hat{N}_{\phi} \equiv \sum_{\mathbf{r}, a, \alpha} \phi_{\mathbf{r}, a, \alpha}^{\dagger} \phi_{\mathbf{r}, a, \alpha} .
$$

and therefore

$$
\begin{aligned}
& \hat{\Omega}(g, h) \phi_{\mathbf{r}, a, \alpha} \hat{\Omega}^{-1}(g, h)=\omega(g, h) \phi_{\mathbf{r}, a, \alpha}, \\
& \left\{\omega(g, h)= \pm 1 \mid g, h \in G_{s}\right\} \in \mathcal{H}^{2}\left(G_{s}, Z_{2}\right) .
\end{aligned}
$$

Clearly operations within IGG commute with all symmetry implementations $\left\{\hat{U}_{g} \mid g \in G_{s}\right\}$ on partons

$$
\left[\hat{\Omega}(g, h), \hat{U}_{g^{\prime}}\right]=0, \quad \forall g, h, g^{\prime} \in G_{s} .
$$

In this case, the projective symmetry implementations $\left\{\hat{U}_{g} \mid g \in G_{s}\right\}$ on partons $\left\{\phi_{\mathbf{r}, a, \alpha}\right\}$ form a central extension of symmetry group $G_{s}$. Different symmetric $Z_{2}$ spin liquids preserving the same symmetry group $G_{s}$ are hence classified by different \pm 1 -valued phase factor sets $\left\{\omega(g, h)= \pm 1 \mid g, h \in G_{s}\right\}$, which mathematically corresponds to the classification of 2nd group cohomology $\mathcal{H}^{2}\left(G_{s}, Z_{2}\right)$ as in 12 . These phase factors are also subject to associativity

$$
\omega(g, h) \omega(f, g h)=\omega(f g, h) \omega(f, g)
$$

They are well-defined only up gauge transformations

$$
\omega(g, h) \rightarrow \omega(g, h) \frac{w_{g h}}{w_{g} \cdot w_{h}}, \quad w_{g}, w_{h}, w_{g h}= \pm 1 .
$$

Two sets of phase factors related by a gauge transformation belong to the same fractionalization class and 
describe the same $Z_{2}$ spin liquid. Therefore gaugeinequivalent phase factor sets $\{\omega(g, h)= \pm 1 \mid g, h \in$ $\left.G_{s}\right\}$ correspond to different fractionalization classes, and hence distinct symmetric $Z_{2}$ spin liquids.

If all phase factors can be chosen to be trivial i.e. $\omega(g, h) \equiv 1$, then the physical symmetry group $G_{s}$ is implemented faithfully on partons. On other other hand, if it is impossible to trivialize all phase factors by any gauge transformation, there exists at least two elements $g, h \in G_{s}$ such that $U_{g} \cdot U_{h}=$ $(-1)^{\hat{N}_{\phi}} \cdot U_{g h}$. These symmetries $g, h$ act projectively on spinons/partons $\left\{\phi_{\mathbf{r}, a, \alpha}\right\}$, and this phenomenon is coined "symmetry fractionalization" "10|14|16 in quantum spin liquids. For gapped symmetric topological orders39, different symmetry fractionalization classes of anyon excitations therein can be fully classified in an abstract setup under the framework of unitary modular tensor category $14 \mid 16$. The parton construction discussed here not only provides a physical manifestation for the abstract mathematical classification, but also applies to gapless $Z_{2}$ spin liquids ${ }^{1920}$ which are beyond the current categorical framework.

Below we list a few gauge-invariant phases, whose different values will correspond to distinct symmetric $Z_{2}$ spin liquids. Each gauge-invariant phase is typically associated with a series of symmetry operations which yields the identity operation $e$ in the symmetry group $G_{s}$, as shown in the left column of TABLE IIIII.

For time reversal symmetry $\boldsymbol{T}$ we have

$$
\boldsymbol{T}^{2}=(-1)^{\hat{N}_{\phi}} \Longrightarrow \omega_{\boldsymbol{T}} \equiv \omega(\boldsymbol{T}, \boldsymbol{T})=-1
$$

since partons/spinons are all Kramers doublets. $e$ is the identity element of symmetry group $G_{s}$.

For mirror reflection symmetry $\boldsymbol{\sigma}$ we have

$$
\sigma^{2}=e \Longrightarrow \omega_{\sigma} \equiv \omega(\sigma, \sigma)= \pm 1
$$

For $n$-fold rotational symmetry $C_{n}$ we have

$$
\left(C_{n}\right)^{n}=e \Longrightarrow \omega_{C_{n}} \equiv \prod_{i=1}^{n-1} \omega\left(C_{n},\left(C_{n}\right)^{i}\right)= \pm 1 \text {. }
$$

With both time reversal and mirror reflection, we have

$$
\boldsymbol{\sigma}^{-1} \boldsymbol{T}^{-1} \boldsymbol{\sigma} \boldsymbol{T}=e \Longrightarrow \omega_{\sigma \boldsymbol{T}} \equiv \frac{\omega(\boldsymbol{\sigma}, \boldsymbol{T})}{\omega(\boldsymbol{T}, \boldsymbol{\sigma})}= \pm 1
$$

These gauge invariant phase factors will be used in following discussions on the gapless criteria and applications to $Z_{2}$ spin liquids on various lattices.

\section{Spinon pair superfluid description of $Z_{2}$ spin liquids}

In the following, we setup the most generic parton Hamiltonian $\hat{H}_{\text {parton }}$ for a symmetric $Z_{2}$ spin liquid, in the framework of parton construction (1). The symmetry group $G_{s}$ considered here includes at least $U(1)$ spin rotational symmetry (say, along $\hat{z}$-axis), time-reversal symmetry $\boldsymbol{T}$, and certain space group symmetries:

$$
G_{s} \supset U(1)_{\mathbf{S}^{z}} \times Z_{2}^{\boldsymbol{T}} \times \text { Space group }
$$

We do not consider spin-orbit couplings in the system, so the space group symmetries considered here are pure spatial operations involving no spin rotations.

Under time reversal operation $\hat{\boldsymbol{T}}=U_{\boldsymbol{T}} \cdot \mathcal{K}$ where $\mathcal{K}$ represents complex conjugation, the partons/spinons transform as spin-1/2 Kramers doublets by construction in (1):

$$
\hat{\boldsymbol{T}} \phi_{\mathbf{r}, a, \alpha} \hat{\boldsymbol{T}}^{-1}=\sum_{\beta} \mathrm{i}\left(\sigma_{y}\right)_{\alpha, \beta} \phi_{\mathbf{r}, a, \beta}
$$

Under $U(1)_{\mathbf{S} z}$ spin rotation they transform as

$$
e^{\mathrm{i} \theta \hat{\mathbf{S}}^{z}} \phi_{\mathbf{r}, a, \alpha} e^{-\mathrm{i} \theta \hat{\mathbf{S}}^{z}}=\sum_{\beta} e^{\mathrm{i} \frac{\theta}{2}\left(\sigma_{z}\right)_{\alpha, \beta}} \phi_{\mathbf{r}, a, \beta}
$$

The conservation of $\hat{z}$-component of total spins makes the Nambus basis $\psi_{\mathbf{r}} \equiv\left(\phi_{\mathbf{r}, a, \uparrow} \phi_{\mathbf{r}, a, \downarrow}^{\dagger}\right)^{T}$ a convenient choice to diagonalize the parton Hamiltonian. Most generally, the symmetry-allowed parton Hamiltonian of a spin- $S$ system can be written in the Nambu basis as

$$
\begin{gathered}
\hat{H}_{\text {parton }}=\hat{H}_{M F}+\hat{H}_{i n t}, \\
\hat{H}_{M F}=\sum_{\mathbf{r}, \mathbf{r}^{\prime}} \psi_{\mathbf{r}}^{\dagger}\left\langle\mathbf{r} \mid \mathbf{r}^{\prime}\right\rangle \psi_{\mathbf{r}^{\prime}}, \quad \psi_{\mathbf{r}} \equiv\left(\begin{array}{c}
\phi_{\mathbf{r}, a, \uparrow} \\
\phi_{\mathbf{r}, a, \downarrow}^{\dagger}
\end{array}\right) .
\end{gathered}
$$

where $\hat{H}_{M F}$ represents the quadratic part at mean-field level and $\hat{H}_{\text {int }}$ for generic symmetry-preserving interactions between partons. The $(4 S) \times(4 S)$ matrices $\left\langle\mathbf{r} \mid \mathbf{r}^{\prime}\right\rangle=\left\langle\mathbf{r}^{\prime} \mid \mathbf{r}\right\rangle^{\dagger}$ are mean-field amplitudes for spinons. The spin rotational symmetry 22 is nothing but the global phase rotation in the Nambu basis:

$$
e^{\mathrm{i} \theta \hat{\mathbf{S}}^{z}} \psi_{\mathbf{r}} e^{-\mathrm{i} \theta \hat{\mathbf{S}}^{z}}=e^{\mathrm{i} \frac{\theta}{2}} \psi_{\mathbf{r}}
$$

while time reversal symmetry (TRS) plays the role of an anti-unitary particle-hole symmetry on the Nambu spinor:

$$
\begin{gathered}
\hat{\boldsymbol{T}} \psi_{\mathbf{r}} \hat{\boldsymbol{T}}^{-1}=\left(\begin{array}{c}
\phi_{\mathbf{r}, a, \downarrow} \\
-\phi_{\mathbf{r}, a, \uparrow}^{\dagger}
\end{array}\right)=\mathrm{i} \tau_{y} \psi_{\mathbf{r}}^{*}, \\
\hat{\boldsymbol{T}}^{2}=(-1)^{\hat{N}_{\phi}}=(-1)^{2 N S+\hat{F}}, \quad \hat{F} \equiv \sum_{\mathbf{r}} \psi_{\mathbf{r}}^{\dagger} \psi_{\mathbf{r}} .
\end{gathered}
$$

where $\vec{\tau}$ are Pauli matrices for the Nambu index, and $N$ is the total number of lattice sites.

For a symmetric $Z_{2}$ spin liquid, the time-reversalsymmetric pair superfluid Hamiltonian in the partons/spinon $\left\{\phi_{\mathbf{r}}\right\}$ basis is mapped to a Bloch Hamiltonian of particle-hole-symmetric insulator in the Nambu basis $\left\{\psi_{\mathbf{r}}\right\}$. Due to $U(1)_{\mathbf{S} z}$ spin conservation, the Nambu particles $\left\{\psi_{\mathbf{r}}\right\}$ have a conserved particle number $\hat{F}$ as defined in 25. Although usually $(-1)^{\hat{F}}$ is used to denote 
fermion parity, here we use it for both (hard-core) Bose and Fermi statistics of partons $\left\{\phi_{\mathbf{r}, a, \alpha}\right\}$. It is instructive to compare the vacua and ground states in these two different basis. Denoting the Fock vacuum of partons $\left\{\phi_{\mathbf{r}}\right\}$ as $|0\rangle$ with

$$
\phi_{\mathbf{r}, a, \alpha}|0\rangle=0, \quad \forall \mathbf{r}, a, \alpha .
$$

the vacuum $\mid$ empty $\rangle$ in the Nambu basis is given by

$$
\begin{aligned}
&\left.\psi_{\mathbf{r}} \mid \text { empty }\right\rangle=0 \Longrightarrow \\
&\mid \text { empty }\rangle=\left(\prod_{\mathbf{r}, a} \phi_{\mathbf{r}, a, \downarrow}^{\dagger}\right)|0\rangle=|-S,-S, \cdots\rangle
\end{aligned}
$$

This is nothing but the fully polarized state where all spins point to negative $\hat{z}$-direction. Now that partons $\left\{\psi_{\mathbf{r}}\right\}$ (or $\left.\left\{\phi_{\mathbf{r}}\right\}\right)$ are either hard-core bosons or fermions, there is also a fully filled state in the Nambu basis

$$
\begin{aligned}
\psi_{\mathbf{r}}^{\dagger}|f u l l\rangle & \left.=0 \Longrightarrow|f u l l\rangle=\left(\prod_{\mathbf{r}, a} \psi_{\mathbf{r}, a, 1}^{\dagger} \psi_{\mathbf{r}, a, 2}^{\dagger}\right) \mid \text { empty }\right\rangle \\
& =\prod_{\mathbf{r}, a} \phi_{\mathbf{r}, a, \downarrow}^{\dagger}|0\rangle=|+S,+S, \cdots\rangle .
\end{aligned}
$$

It is the other polarized state where all spins point to the positive $\hat{z}$-direction, and the time reversal partner of the Nambu vacuum

$$
|f u l l\rangle=\hat{\boldsymbol{T}} \mid \text { empty }\rangle .
$$

It's straightforward to show that no matter partons $\left\{\psi_{\mathbf{r}}\right\}$ are either hard-core bosons or fermions, the Nambu particle number $\hat{F}$ in 25 is related to the total spin $\hat{\mathbf{S}}^{z}$ by

$$
\hat{2} \mathbf{S}^{z} \equiv \sum_{\mathbf{r}, a}\left(\phi_{\mathbf{r}, a, \uparrow}^{\dagger} \phi_{\mathbf{r}, a, \uparrow}-\phi_{\mathbf{r}, a, \downarrow}^{\dagger} \phi_{\mathbf{r}, a, \downarrow}\right)=\hat{F}-2 N S
$$

Consequently, TRS 25 enforces the Nambu particle number $\hat{F}$ to be at "half filling":

$$
\mathbf{S}^{z}=0 \Longrightarrow \frac{\hat{F}}{N}=\frac{1}{N} \sum_{\mathbf{r}} \psi_{\mathbf{r}}^{\dagger} \psi_{\mathbf{r}}=2 S=\text { odd }
$$

Hereafter we'll stick to the Nambu basis which allows us to make the best of $U(1)_{\mathbf{S}} z$ symmetry.

A generic time-reversal-symmetric $Z_{2}$ spin liquid can be obtained by projecting the following parton state

$$
\left.\left|\Psi_{\text {parton }}\right\rangle=\sum_{\left\{\mathbf{r}_{i}, a_{i}, b_{i}\right\}} \Psi\left(\left\{\mathbf{r}_{i}, a_{i}, b_{i}\right\}\right) \prod_{i=1}^{N S} \psi_{\mathbf{r}_{i}, a_{i}, 1}^{\dagger} \psi_{\mathbf{r}_{i}, b_{i}, 2}^{\dagger} \mid \text { empty }\right\rangle
$$

which satisfies

$$
\mathbf{S}^{z}\left|\Psi_{\text {parton }}\right\rangle=0
$$

apart from one exception described below. In a system with $N=$ odd half-integer spins, Kramers theorem dictates at least two-fold degeneracy for all eigenstates. Indeed the parton state 32 will not be well-defined since
$N S$ is a half integer. In this case, the parton ground states typically create $N S \pm \frac{1}{2}$ pairs of Nambu particles on top of Nambu vacuum $|e m p t y\rangle$, with a total spin $\mathbf{S}^{z}= \pm \frac{1}{2}$

Next we analyze the symmetry implementations on the Nambu vacuum $|e m p t y\rangle$ and parton state $\left|\Psi_{\text {parton }}\right\rangle$. Since the Nambu vacuum $|e m p t y\rangle$ in 27) is a physical state where all spins are fully polarized along negative $\hat{z}$ direction, symmetries must act faithfully on the Nambu vacuum 54 i.e.

$$
\hat{U}_{g} \cdot \hat{U}_{h}|e m p t y\rangle=\hat{U}_{g h}|e m p t y\rangle, \forall g, h \in G_{s} .
$$

with the only exception that

$$
\left.\left.\hat{\boldsymbol{T}}^{2} \mid \text { empty }\right\rangle=(-1)^{2 N S} \mid \text { empty }\right\rangle .
$$

associated with Kramers degeneracy if $2 N S=$ odd. In other words, the series of combined symmetry operations on the left column of TABLEIIIII all act trivially on the Nambu vacuum (27), except for $\boldsymbol{T}^{2}=\boldsymbol{e}$.

On an arbitrary parton state $\left|\Psi_{\text {parton }}\right\rangle$ in $(32)$, symmetry operations act in the following way

$$
\hat{U}_{g} \cdot \hat{U}_{h}\left|\Psi_{\text {parton }}\right\rangle=[\omega(g, h)]^{\hat{F}} \cdot \hat{U}_{g h}\left|\Psi_{\text {parton }}\right\rangle
$$

or equivalently

$$
\hat{U}_{g} \cdot \hat{U}_{h}=[\omega(g, h)]^{\hat{F}} \cdot \hat{U}_{g h}
$$

This projective symmetry operation is crucial for the gapless criteria for $Z_{2}$ spin liquids as we will show below.

\section{CRITERIA FOR SYMMETRY PROTECTED GAPLESS $Z_{2}$ SPIN LIQUIDS}

Previously we have setup the parton Hamiltonian of a generic symmetric pair superfluid, which describes a symmetric $Z_{2}$ spin liquid in the parton construction (1), irrespective of the (hard-core)Bose or Fermi statistics of partons. An important property of a $Z_{2}$ spin liquid is whether there is a finite energy gap in the excitation spectrum or not. Usually the existence of an excitation gap depends on the specified Hamiltonian of the system. However in certain quantum spin liquids, one can sometimes rule out the possibility of an energy gap once the physical Hilbert space and symmetry implementations on spinons are specified 40 42, without referring to any specific Hamiltonian.

Here we will address the following question in the context of symmetric $Z_{2}$ spin liquids: given a lattice system of half-integer spins with certain space group symmetries, for a specific symmetry fractionalization class of spinons, is it possible to construct a gapped symmetric $Z_{2}$ spin liquid? Always assuming time reversal symmetry 21 and $U(1)$ spin rotational symmetry 22 , we consider all possible fractionalization classes of crystal symmetry on spinons in a $Z_{2}$ spin liquid (see e.g. TABLE IIIII). We 
do not specify the statistics of spinons $\left\{\phi_{\mathbf{r}, a, \alpha}\right\}$, i.e. they can be either hard-core bosons or fermions. If the generic parton/spinon Hamiltonian (23) cannot support a unique gapped ground state, our parton construction will lead to a gapless $Z_{2}$ spin liquid, whose low-energy physics are described by gapless spinons coupled to dynamical $Z_{2}$ gauge fields. Previously, gapless $Z_{2}$ spin liquids were mostly discussed at the mean-field leve $19 \mid 20$ in the parton construction, but it is not clear whether interactions between spinons or gauge fluctuations can open up a gap in the spectrum. Although gapless $Z_{2}$ spin liquids were found numerically in projected wavefunctions 43144 on the square lattice, the stability of these states with respect to general perturbations remains an open issue. Here using the conserved particle number $\hat{F}$ in the Nambu basis, as a consequence of $U(1)_{\mathbf{s}} z$ symmetry, we are able to non-perturbatively prove the stability of certain gapless $Z_{2}$ spin liquids with respect to any symmetry-preserving perturbations, including arbitrary interactions between spinons.

In the following we establish three criteria on the symmetry fractionalization class of spinons, which lead to a gapless spectrum of the interacting parton/spinon Hamiltonian 23. These criteria dictate the stability of gapless $Z_{2}$ spin liquids associated with certain spinon fractionalization class $\left\{\omega(g, h) \mid g, h \in G_{s}\right\} \in \mathcal{H}^{2}\left(G_{s}, Z_{2}\right)$. Independent of whether partons/spinons are hard-core bosons or fermions, we take the following strategy to establish the gapless criteria. Given certain spinon symmetry fractionalization class $\left\{\omega(g, h) \mid g, h \in G_{s}\right\}$, we first prove symmetry-enforced zero-energy degeneracy, at the level of bilinear mean-field Ansatz $\hat{H}_{M F}$ of partons/spinons. Next we go beyond mean-field Ansatz, and argue the impossibility of a unique gapped many-body ground state for the interacting parton/spinon Hamiltonian (23), using the Schmidt decomposition of a shortrange-entangled (gapped) state 25 .

\section{A. Two perpendicular mirror planes intersecting at one half-integer spin}

The first criterion comes from two perpendicular mirror reflection planes, which intersect at one lattice site with a half-integer spin on it. Take square lattice for example, we consider two perpendicular mirror planes $\boldsymbol{\sigma}$ and $R_{x}$ in FIG. 1. Consider the following projective symmetry implementation on spinons

$$
\begin{gathered}
\hat{\boldsymbol{\sigma}} \hat{R}_{x} \hat{\boldsymbol{\sigma}}^{-1} \hat{R}_{x}^{-1}=\boldsymbol{e} \Longrightarrow \\
\frac{\omega\left(\boldsymbol{\sigma}, R_{x}\right)}{\omega\left(R_{x}, \boldsymbol{\sigma}\right)}=-1 \Leftrightarrow \hat{U}_{\boldsymbol{\sigma}} \hat{U}_{R_{x}} \hat{U}_{\boldsymbol{\sigma}}^{-1} \hat{U}_{R_{x}}^{-1}=(-1)^{\hat{F}} .
\end{gathered}
$$

where $\hat{F}=\sum_{\mathbf{r}} \psi_{\mathbf{r}}^{\dagger} \psi_{\mathbf{r}}$ is the parton number in the Nambu basis. Since the combination of the two mirror reflections is nothing but the two-fold rotation $C_{2}=\sigma R_{x}$ around

\begin{tabular}{|c|c|c|c|}
\hline Algebraic Identity & $\begin{array}{l}\text { SB } b_{\alpha} \text { in } \\
\text { Ref. [32 }\end{array}$ & $\begin{array}{l}\text { AF } f_{\alpha} \text { in } \\
\text { Ref. [19 }\end{array}$ & $\begin{array}{c}\text { fractionalization } \\
\text { class of spinons }\end{array}$ \\
\hline$T_{2}^{-1} T_{1}^{-1} T_{2} T_{1}=\boldsymbol{e}$ & $(-1)^{p_{1}}$ & $\eta_{x y}$ & $\omega_{T_{1} T_{2}}$ \\
\hline $\boldsymbol{\sigma}^{-1} T_{1} \boldsymbol{\sigma} T_{1}^{-1}=\boldsymbol{e}$ & $(-1)^{p_{2}}$ & $\eta_{x p y}$ & $\omega_{\boldsymbol{\sigma} T_{1}}$ \\
\hline$\left(\boldsymbol{\sigma} T_{2}\right)^{2}=\boldsymbol{e}$ & $(-1)^{p_{3}+p_{4}}$ & $\eta_{\boldsymbol{\sigma}} \eta_{x p x}$ & $\omega_{\boldsymbol{\sigma} T_{2}} \omega_{\boldsymbol{\sigma}}$ \\
\hline$C_{4}{ }^{-1} T_{1} C_{4} T_{2}=\boldsymbol{e}$ & 1 & 1 & 1 \\
\hline$C_{4}{ }^{-1} T_{2} C_{4} T_{1}^{-1}=\boldsymbol{e}$ & $(-1)^{p_{2}+p_{3}}$ & $\eta_{x p y} \eta_{x p x}$ & $\omega_{\boldsymbol{\sigma} T_{1}} \omega_{\boldsymbol{\sigma} T_{2}}$ \\
\hline $\boldsymbol{\sigma}^{2}=\boldsymbol{e}$ & $(-1)^{p_{4}}$ & $\eta_{\boldsymbol{\sigma}}$ & $\omega_{\boldsymbol{\sigma}}$ \\
\hline$R_{x y}^{2}=\left(C_{4} \boldsymbol{\sigma}\right)^{2}=\boldsymbol{e}$ & $(-1)^{p_{4}+p_{7}}$ & $\eta_{\boldsymbol{\sigma}} \eta_{\boldsymbol{\sigma} C_{4}}$ & $\omega_{R}$ \\
\hline$\left(C_{4}\right)^{4}=\boldsymbol{e}$ & 1 & $\eta_{C_{4}}$ & $\omega_{C_{4}}$ \\
\hline$T_{1}^{-1} \boldsymbol{T}^{-1} T_{1} \boldsymbol{T}=\boldsymbol{e}$ & $(-1)^{p_{8}}$ & $\eta_{t}$ & $\omega_{T_{1} \boldsymbol{T}}$ \\
\hline$T_{2}^{-1} \boldsymbol{T}^{-1} T_{2} \boldsymbol{T}=\boldsymbol{e}$ & $(-1)^{p_{8}}$ & $\eta_{t}$ & $\omega_{T_{1} \boldsymbol{T}}$ \\
\hline $\boldsymbol{\sigma}^{-1} \boldsymbol{T}^{-1} \boldsymbol{\sigma} \boldsymbol{T}=\boldsymbol{e}$ & $(-1)^{p_{4}}$ & $\eta_{\boldsymbol{\sigma} \boldsymbol{T}}$ & $\omega_{\boldsymbol{\sigma} \boldsymbol{T}}$ \\
\hline$R_{x y}^{-1} \boldsymbol{T}^{-1} R_{x y} \boldsymbol{T}=\boldsymbol{e}$ & $(-1)^{p_{4}+p_{7}}$ & $\eta_{C_{4} \boldsymbol{T}} \eta_{\boldsymbol{\sigma} \boldsymbol{T}}$ & $\omega_{R \boldsymbol{T}}$ \\
\hline $\boldsymbol{T}^{2}=\boldsymbol{e}$ & -1 & -1 & -1 \\
\hline
\end{tabular}

Table I: Square lattice: spinon symmetry fractionalization class $\left(\mathbb{Z}_{2}\right)^{9} \subset \mathcal{H}^{2}\left(P 4 g m \times Z_{2}^{T}, \mathbb{Z}_{2}\right)$ for $Z_{2}$ spin liquids of halfinteger spins, and their realizations in $S=\frac{1}{2}$ Schwinger-boson (SB) and Abrikosov-fermion (AF) representations, following the notation of Ref. 1932 The fractionalization classes from 2nd group cohomology ${ }^{10}$ are characterized by nine $Z_{2}$-valued gauge-invariant phases $\omega= \pm 1$. We show that a $Z_{2}$ spin liquid is gapless if violating any of the following conditions: $\omega_{\sigma}=\omega_{\sigma \boldsymbol{T}}, \omega_{R}=\omega_{R T}$ and $\omega_{C_{4}}=1$. This leads to only $2^{6}=64$ distinct gapped $Z_{2}$ spin liquids, all realized in both $\mathrm{SB}^{32}$ and $\mathrm{AF}^{[19}$ representations. There are also 96 symmetry protected gapless $Z_{2}$ spin liquids in the AF representation.

their intersection site, the above gauge-invariant phase factor can also be written as

$$
\frac{\omega\left(\boldsymbol{\sigma}, R_{x}\right)}{\omega\left(R_{x}, \boldsymbol{\sigma}\right)}=\frac{\omega\left(\boldsymbol{\sigma} R_{x}, \boldsymbol{\sigma} R_{x}\right)}{\omega\left(R_{x}, R_{x}\right) \cdot \omega(\boldsymbol{\sigma}, \boldsymbol{\sigma})} \equiv \frac{\omega_{C_{2}}}{\omega_{R_{x}} \cdot \omega_{\boldsymbol{\sigma}}}=-1
$$

First it's straightforward to show that in mean-field Ansatz, each single-particle level of partons/spinons must be at least 2 -fold degenerate. The $4 N S \times 4 N S$ mean-field Ansatz Hamiltonian can be diagonalized as

$$
\hat{H}_{M F}=\sum_{\mathbf{r}, \mathbf{r}^{\prime}} \psi_{\mathbf{r}}^{\dagger}\left\langle\mathbf{r} \mid \mathbf{r}^{\prime}\right\rangle \psi_{\mathbf{r}^{\prime}}=\sum_{E} E \gamma_{E}^{\dagger} \gamma_{E}
$$

where $E$ represents the single-particle eigen-energy, and $\gamma_{E}$ is the annihilation operator of the associated eigenstate. TRS in (25) as an anti-unitary particle-hole symmetry indicates that single-spinon levels always show up in pairs with opposite energies:

$$
\hat{\boldsymbol{T}} \gamma_{E} \hat{\boldsymbol{T}}^{-1}=\gamma_{-E}^{\dagger}
$$

As a result, the number of positive and negative singlespinon energy levels must both equal $2 N S$.

In the presence of mirror reflection symmetry $\boldsymbol{\sigma}$, each eigenmode can be labeled by their $\boldsymbol{\sigma}$ eigenvalue $q_{E}(\boldsymbol{\sigma})$ :

$$
\begin{gathered}
\hat{U}_{\boldsymbol{\sigma}} \gamma_{E} \hat{U}_{\boldsymbol{\sigma}}^{-1}=q_{E}(\boldsymbol{\sigma}) \gamma_{E}, \\
{\left[q_{E}(\boldsymbol{\sigma})\right]^{2}=\omega_{\boldsymbol{\sigma}}= \pm 1 .}
\end{gathered}
$$




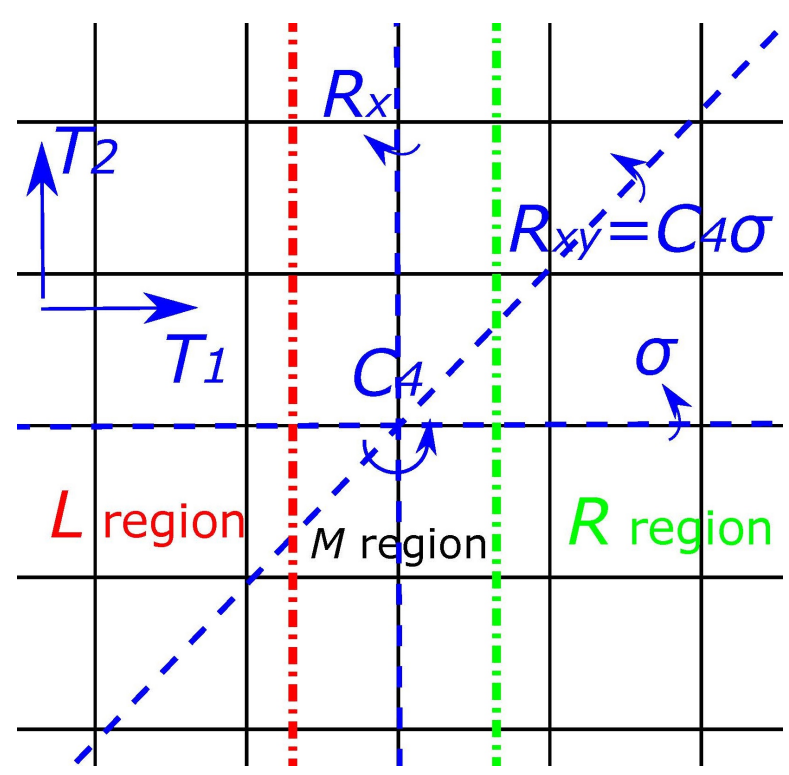

Figure 1: (color online) Space group ( $P 4 \mathrm{gm})$ symmetries of square lattice, generated by two translations $T_{1,2}$, mirror reflection $\sigma$ and 4 -fold rotation $C_{4}$. Two other mirror reflections are defined as $R_{x y}=C_{4} \boldsymbol{\sigma}$ and $R_{x}=\left(C_{4}\right)^{2} \boldsymbol{\sigma}$. Red and green dotted dash lines denote two entanglement cuts of the lattice, related by mirror $R_{x}$ or inversion $I \equiv C_{2}=\left(C_{4}\right)^{2}$.

On the other hand, mirror reflection $R_{x}$ satisfying (38) leads to another degenerate single-spinon level $\hat{U}_{R_{x}} \gamma_{E} \hat{U}_{R_{x}}^{-1}$ with an opposite $\boldsymbol{\sigma}$ eigenvalue $-q_{E}(\boldsymbol{\sigma})$ since

$$
\begin{gathered}
\hat{U}_{\boldsymbol{\sigma}}\left(\hat{U}_{R_{x}} \gamma_{E} \hat{U}_{R_{x}}^{-1}\right) \hat{U}_{\boldsymbol{\sigma}}^{-1}=-\hat{U}_{R_{x}} \hat{U}_{\boldsymbol{\sigma}} \gamma_{E} \hat{U}_{\boldsymbol{\sigma}}^{-1} \hat{U}_{R_{x}}^{-1} \\
=-q_{E}(\boldsymbol{\sigma}) \cdot\left(\hat{U}_{R_{x}} \gamma_{E} \hat{U}_{R_{x}}^{-1}\right)
\end{gathered}
$$

Therefore each single-spinon level must be at least 2-fold degenerate, due to anti-commuting mirror operations $\hat{U}_{\boldsymbol{\sigma}}$ and $\hat{U}_{R_{x}}$ on spinons as shown in (38).

Now consider a lattice with $N=$ odd number of lattice sites with one half-integer spin- $S$ on each site, such as a odd by odd square lattice on FIG. 1. Since the number of both positive and negative energy eigenstates is $2 N S=$ odd, and each energy level is at least two-fold degenerate, there must be (at least) two degenerate zeroenergy eigenstates in the single-spinon spectrum. Therefore at mean-field level, the spinon/parton ground state must have at least 2-fold degeneracy.

The above argument can be easily generalized to interacting parton/spinon Hamiltonian (23) on an $N=$ odd lattice. Since in the Nambu basis, the many-body ground state $\left|\Psi_{\text {parton }}\right\rangle$ has a conserved particle number $\hat{F}=$ $2 N S=$ odd, two mirror reflections $\hat{U}_{\boldsymbol{\sigma}}$ and $\hat{U}_{R_{x}}$ anticommute on the many-body ground state as shown in (37), leading to at least 2-fold degeneracy. However, there are some subtlety in defining a $Z_{2}$ spin liquid with $N=$ odd half-integer spins, where the physical many-spin ground state contains an odd number of spinons and must be at least 2-fold degenerate due to Kramers theorem. Below we put previous intuitive arguments on a firmer ground, by using Schmidt decomposition of the manyspinon ground state on an infinite cylinder 27 .

Consider interacting spinon Hamiltonian 23 on an infinite cylinder wrapped along $\hat{y}$ direction in FIG. 1 . where the cylinder circumference is $L_{y}=$ odd. Assume it hosts a unique gapped ground state $\left|\Psi_{\text {parton }}\right\rangle$, preserving mirror reflections $R_{x}, \boldsymbol{\sigma}$ and time reversal symmetry $\boldsymbol{T}$. This gapped ground state is also a short-range-entangled (SRE) $\operatorname{state}^{26}$ on this infinite cylinder. Across the $\boldsymbol{\sigma}$ preserving entanglement cut denoted by the red dotteddash line in FIG. 1. the SRE ground state can be Schmidt decomposed into

$$
\left|\Psi_{\text {parton }}\right\rangle=\sum_{\nu} w_{\nu}|\nu\rangle_{L} \otimes|\nu\rangle_{\bar{L}}
$$

where $w_{\nu}$ are Schmidt weights. $\left\{|\nu\rangle_{L}\right\}$ and $\left\{|\nu\rangle_{\bar{L}}\right\}$ denote a set of orthonormal basis for the Hilbert space of region $L$ and $\bar{L}$. The Schmidt spectrum $\left\{w_{\nu}\right\}$ is discrete for SRE state $\left|\Psi_{\text {parton }}\right\rangle$. Since the bipartition (red line) of the lattice preserves mirror $\sigma$, the orthonormal basis can be labeled by their reflection quantum number $q(\boldsymbol{\sigma})$ :

$$
\hat{U}_{\boldsymbol{\sigma}}|\nu\rangle_{L}=q_{\nu, L}(\boldsymbol{\sigma})|\nu\rangle_{L}, \quad \hat{U}_{\boldsymbol{\sigma}}|\nu\rangle_{\bar{L}}=q_{\nu, \bar{L}}(\boldsymbol{\sigma})|\nu\rangle_{\bar{L}}
$$

which must satisfy

$$
q_{\nu, L}(\boldsymbol{\sigma}) q_{\nu, \bar{L}}(\boldsymbol{\sigma}) \equiv q_{\boldsymbol{\sigma}}= \pm 1, \quad \forall \nu .
$$

so that parton/spinon ground state $\left|\Psi_{\text {parton }}\right\rangle$ preserves mirror $\boldsymbol{\sigma}$ with eigenvalue $q_{\boldsymbol{\sigma}}$.

Now let's consider another entanglement cut related to the previous one by mirror reflection $R_{x}$, depicted by the green dotted-dash line in FIG. 1. The Schmidt decomposition across this cut is given by

$$
\left|\Psi_{\text {parton }}\right\rangle=\sum_{\nu} w_{\nu}^{\prime}|\nu\rangle_{R} \otimes|\nu\rangle_{\bar{R}},
$$

where by mirror reflection we can choose

$$
w_{\nu}^{\prime}=w_{\nu}, \quad|\nu\rangle_{R}=\hat{U}_{R_{x}}|\nu\rangle_{L}, \quad|\nu\rangle_{\bar{R}}=\hat{U}_{R_{x}}|\nu\rangle_{\bar{L}} .
$$

Note that on an infinite cylinder, the total particle number $\hat{F}_{L}=\sum_{\mathbf{r} \in L} \psi_{\mathbf{r}}^{\dagger} \psi_{\mathbf{r}}$ on the l.h.s. of an entanglement cut is ill-defined in the thermodynamic limit 27 , however the charge fluctuation around its average value

$$
\hat{Q}_{L} \equiv \sum_{\mathbf{r} \in L}\left(\psi_{\mathbf{r}}^{\dagger} \psi_{\mathbf{r}}-2 S\right)=-\hat{Q}_{\bar{L}} \in \mathbb{Z},
$$

is still well-defined. Hence we have

$$
\begin{gathered}
(-1)^{\hat{F}_{L}}|\nu\rangle_{L}=e^{\mathrm{i} \Phi}(-1)^{Q_{\nu, L}}|\nu\rangle_{L}, \quad \forall \nu, \\
\hat{Q}_{L}|\nu\rangle_{L}=Q_{\nu, L}|\nu\rangle_{L}, \\
(-1)^{\hat{F}_{\bar{L}}}|\nu\rangle_{\bar{L}}=e^{-\mathrm{i} \Phi}(-1)^{Q_{\nu, \bar{L}}}|\nu\rangle_{\bar{L}}, \quad \forall \nu, \\
\hat{Q}_{\bar{L}}|\nu\rangle_{\bar{L}}=Q_{\nu, \bar{L}}|\nu\rangle_{\bar{L}}
\end{gathered}
$$


where $\Phi$ is an ambiguous but $\nu$-independent phase 27 . By mirror symmetry $R_{x}$ it's also clear that

$$
Q_{\nu, R}=Q_{\nu, L}=-Q_{\nu, \bar{L}}=-Q_{\nu, \bar{R}} \in \mathbb{Z}
$$

Making use of symmetry action (38), it's straightforward to work out the mirror $\boldsymbol{\sigma}$-eigenvalues of Schmidt components as

$$
\begin{gathered}
\hat{U}_{\boldsymbol{\sigma}}|\nu\rangle_{R}=e^{\mathrm{i} \Phi}(-1)^{Q_{\nu, L}} q_{\nu, L}(\boldsymbol{\sigma})|\nu\rangle_{R}, \\
\hat{U}_{\boldsymbol{\sigma}}|\nu\rangle_{\bar{R}}=e^{-\mathrm{i} \Phi}(-1)^{Q_{\nu, \bar{L}}} q_{\nu, \bar{L}}(\boldsymbol{\sigma})|\nu\rangle_{\bar{R}}
\end{gathered}
$$

Applying mirror reflection $\boldsymbol{\sigma}$ twice and requiring $q_{\nu, L}\left(\boldsymbol{\sigma}^{2}\right)=q_{\nu, R}\left(\boldsymbol{\sigma}^{2}\right)=1$, we obtain that 25

$$
e^{2 \mathrm{i} \Phi}=1
$$

On the other hand notice that region $\bar{L}=M \bigcup R$ (see FIG. 1), hence the Schmidt components on the r.h.s. of the two cuts are related by addition of region $M$ (a column containing $L_{y}=$ odd sites):

$$
|\nu\rangle_{\bar{L}}=\sum_{\mu, \rho} B_{\mu \nu}^{\rho}|\rho\rangle_{M} \otimes|\mu\rangle_{R}
$$

where $\left\{|\rho\rangle_{M}\right\}$ is a set of orthonormal basis for Hilbert space in region $M$, with particle number satisfying

$$
\begin{gathered}
\hat{F}_{M}|\rho\rangle_{M}=\left(Q_{\rho, M}+L_{y} S\right)|\rho\rangle_{M}, \\
Q_{\nu, \bar{L}}=Q_{\rho, M}+Q_{\mu, R}, \quad \forall B_{\mu \nu}^{\rho} \neq 0 .
\end{gathered}
$$

Now let's perform a series of symmetry operations $\hat{U}_{\boldsymbol{\sigma}} \hat{U}_{R_{x}} \hat{U}_{\boldsymbol{\sigma}}^{-1} \hat{U}_{R_{x}}^{-1}$ on both sides of state (54), and we reach a contradiction to 53

$$
e^{\mathrm{i} \Phi}=(-1)^{L_{y} S} e^{-\mathrm{i} \Phi}
$$

for a $L_{y}=$ odd cylinder. Therefore we've shown that symmetry implementation 38 is incompatible with a unique gapped symmetric ground state $\left|\Psi_{\text {parton }}\right\rangle$ of any interacting parton/spinon Hamiltonian (23). Since the above arguments is valid for any odd circumference $L_{y}=1$ mod 2 of the cylinder, it is naturally applicable to a twodimensional system.

As a result, we have established that for spinon symmetry fractionalization class (38), with two perpendicular mirror planes, time reversal and $U(1)_{\mathbf{S}} z$ spin rotational symmetries, it is impossible to have a gapped symmetric $Z_{2}$ spin liquid. This also proves the stability of gapless symmetric $Z_{2}$ spin liquids protected by spinon fractionalization class 38 .

\section{B. Mirror plane crossing an odd number of half-integer spins}

The 2nd criterion for gapless $Z_{2}$ spin liquids is the following implementation of time reversal $\boldsymbol{T}$ and a mirror reflection (say, $R_{x}$ in FIG. 1), whose mirror planes crosses an odd number of half-integer spins (i.e. odd sites):

$$
\begin{gathered}
\boldsymbol{T} R_{x} \boldsymbol{T}^{-1} R_{x}=\boldsymbol{e} \Longrightarrow \\
\omega_{R_{x}} \boldsymbol{T} \omega_{R_{x}}=-1 \Leftrightarrow \hat{\boldsymbol{T}} \hat{U}_{R_{x}} \hat{\boldsymbol{T}}^{-1} \hat{U}_{R_{x}}=(-1)^{\hat{F}} .
\end{gathered}
$$

This gauge-invariant phase factor of symmetry fractionalization can also be written as

$$
\frac{\omega\left(\boldsymbol{T} R_{x}, \boldsymbol{T} R_{x}\right)}{\omega(\boldsymbol{T}, \boldsymbol{T})}=\frac{\omega\left(\boldsymbol{T}, R_{x}\right)}{\omega\left(R_{x}, \boldsymbol{T}\right)} \cdot \omega\left(R_{x}, R_{x}\right)=\omega_{R_{x}} \boldsymbol{T}_{R_{x}}=-1
$$

First at single-particle level i.e. for the parton meanfield Ansatz (40, we can label single-spinon modes by their mirror eigenvalues $q_{E}\left(R_{x}\right)$ as defined in 42 . Performing time reversal operation on each mode will lead to another mode with the opposite energy

$$
\begin{gathered}
\boldsymbol{T} \gamma_{E} \boldsymbol{T}^{-1}=\gamma_{-E}^{\dagger}, \quad\left|q_{E}\left(R_{x}\right)\right|^{2}=1 \Longrightarrow \\
\hat{U}_{R_{x}} \gamma_{-E}^{\dagger} \hat{U}_{R_{x}}^{-1}=-\boldsymbol{T} \hat{U}_{R_{x}}^{-1} \gamma_{E} \hat{U}_{R_{x}} \boldsymbol{T}^{-1}=-q_{E}\left(R_{x}\right) \gamma_{-E}^{\dagger} \\
\Longrightarrow \hat{U}_{R_{x}} \gamma_{-E} \hat{U}_{R_{x}}^{-1}=-q_{E}^{*}\left(R_{x}\right) \gamma_{-E} .
\end{gathered}
$$

Let's first assume there is a finite energy gap in the singlespinon spectrum, separating positive energy states from those with negative energies. Now that each positive energy level $\gamma_{E}$ always shows up in pairs with its timereversal partner $\gamma_{-E}$, the total mirror eigenvalue of all energy levels is given by

$$
\begin{gathered}
\hat{U}_{R_{x}}\left(\prod_{E} \gamma_{E}\right) \hat{U}_{R_{x}}^{-1}= \\
\prod_{E>0}\left(q_{E}\left(R_{x}\right) \cdot\left[-q_{E}^{*}\left(R_{x}\right)\right]\right)=(-1)^{2 N S} .
\end{gathered}
$$

This leads to a total mirror eigenvalue -1 of all energy levels on a lattice with $N=$ odd sites, which is impossible in any physical parton/spinon Hamiltonian. This contradiction can only be resolved by the existence of zero-energy single-spinon modes, i.e. a gapless spinon spectrum.

Below we prove that a unique SRE symmetric ground state of interacting spinon/parton Hamiltonian 23 is impossible on an infinite $R_{x}$-preserving cylinder with circumference $L_{y}=$ odd (see FIG. 1), again using its Schmidt decomposition. Assuming a unique SRE ground state, again it can be Schmidt decomposed into

$$
\begin{gathered}
\left|\Psi_{\text {parton }}\right\rangle=\sum_{\nu} w_{\nu}|\nu\rangle_{L} \otimes|\nu\rangle_{\bar{L}}=\sum_{\nu} w_{\nu}|\nu\rangle_{R} \otimes|\nu\rangle_{\bar{R}} \\
|\nu\rangle_{R}=\hat{U}_{R_{x}}|\nu\rangle_{L}, \quad|\nu\rangle_{\bar{R}}=\hat{U}_{R_{x}}|\nu\rangle_{\bar{L}}
\end{gathered}
$$

Time reversal operation 25 in the Nambu basis indicates that

$$
\hat{\boldsymbol{T}}^{2}|\nu\rangle_{L}=(-1)^{\sum_{\mathbf{r} \in L}\left(\psi_{\mathbf{r}}^{\dagger} \psi_{\mathbf{r}}-2 S\right)}|\nu\rangle_{L}=(-1)^{Q_{\nu, L}}|\nu\rangle_{L} .
$$

where $Q_{\nu, L}$ are particle number fluctuation in region $L$, defined in (49)-(51). Again since the parton density $2 S \in$ $\mathbb{Z}$ is an integer in the Nambu basis, we have the following relation

$$
e^{2 \mathrm{i} \Phi}=1
$$


for ambiguous phase $\Phi$ in 50 for the Nambu particle parity of Schmidt eigenstates. Due to mirror reflection symmetry $R_{x}$ we have

$$
\begin{aligned}
\hat{\boldsymbol{T}} \hat{U}_{R_{x}} & \hat{\boldsymbol{T}}^{-1} \hat{U}_{R_{x}}|\nu\rangle_{R}=(-1)^{\hat{F}_{R}}|\nu\rangle_{R}=e^{\mathrm{i} \Phi}(-1)^{Q_{\nu, R}}|\nu\rangle_{R} \\
& \hat{\boldsymbol{T}} \hat{U}_{R_{x}} \hat{\boldsymbol{T}}^{-1} \hat{U}_{R_{x}}|\nu\rangle_{\bar{L}}=e^{-\mathrm{i} \Phi}(-1)^{Q_{\nu, \bar{L}}}|\nu\rangle_{\bar{L}}
\end{aligned}
$$

Following the same strategy $(\bar{L}=M \bigcup R)$ we used in proving the 1 st criterion, by acting $\hat{\boldsymbol{T}} \hat{U}_{R_{x}} \hat{\boldsymbol{T}}^{-1} \hat{U}_{R_{x}}$ on both sides of (54) we end up with the following contradiction

$$
e^{-\mathrm{i} \Phi}=(-1)^{L_{y} S} e^{\mathrm{i} \Phi}
$$

on a cylinder with circumference $L_{y}=$ odd.

Therefore we've proved that symmetry fractionalization class (58) of spinons is incompatible with a symmetric SRE ground state for the generic interacting parton/spinon Hamiltonian (23). This therefore leads to stable gapless $Z_{2}$ spin liquids protected by mirror and time reversal symmetries, characterized by spinon symmetry fractionalization (58).

\section{C. $C_{2 n}$ rotation or inversion centered at a half-integer spin}

The 3 rd criterion is provided by the interplay of $2 n$-fold site-centered rotation $C_{2 n}$ and time reversal operation $\boldsymbol{T}$. In particular, a $C_{2 n}$ rotation necessarily imply a two-fold rotation

$$
\hat{C}_{2}=\left(\hat{C}_{2 n}\right)^{n} \equiv \hat{I}
$$

around the same site with an half-integer spin on it. This 2 -fold rotation can also be viewed as the inversion operation $\hat{I}$ for the $2 \mathrm{~d}$ plane. Our 3rd criterion for gapless $Z_{2}$ spin liquids is guaranteed by the following spinon symmetry fractionalization class

$$
\begin{gathered}
\boldsymbol{T} C_{2} \boldsymbol{T}^{-1} C_{2}=e \Longrightarrow \\
\frac{\omega\left(C_{2} \boldsymbol{T}, C_{2} \boldsymbol{T}\right)}{\omega(\boldsymbol{T}, \boldsymbol{T})}=-1 \Leftrightarrow \hat{\boldsymbol{T}} \hat{U}_{C_{2}} \hat{\boldsymbol{T}}^{-1} \hat{U}_{C_{2}}=(-1)^{\hat{F}} .
\end{gathered}
$$

The above symmetry fractionalization class is quite analogous to previous criterion (58) for reflection $\hat{R}_{x}$ and time reversal $\boldsymbol{T}$, where the only difference is to replace mirror $\hat{R}_{x}$ by inversion $\hat{C}_{2}=\hat{I}$. It's straightforward to show that our previous arguments on criterion (58) can be applied completely in parallel to prove the above criterion (67).

The above criterion can be further refined for $2 n$-fold rotation $C_{2 n}$, depending on whether $n$ is an even or odd integer ${ }^{55}$. It will lead to two different criteria for gapless $Z_{2}$ spin liquids protected by $C_{2 n}$ rotations, as we elaborate below.
First of all, due to algebraic equality (66), it's straightforward to verify the following relations for gaugeinvariant phase factors of spinon symmetry fractionalization:

$$
\begin{gathered}
\omega\left(C_{2}, C_{2}\right)=\prod_{i=1}^{2 n-1} \omega\left(C_{2 n},\left(C_{2 n}\right)^{i}\right) \equiv \omega_{C_{2 n}}, \\
\frac{\omega\left(C_{2}, \boldsymbol{T}\right)}{\omega\left(\boldsymbol{T}, C_{2}\right)}=\left[\frac{\omega\left(C_{2 n}, \boldsymbol{T}\right)}{\omega\left(\boldsymbol{T}, C_{2 n}\right)}\right]^{n}
\end{gathered}
$$

Meanwhile criterion (67) is the same as

$$
\frac{\omega\left(C_{2} \boldsymbol{T}, C_{2} \boldsymbol{T}\right)}{\omega(\boldsymbol{T}, \boldsymbol{T})}=\frac{\omega\left(C_{2}, \boldsymbol{T}\right)}{\omega\left(\boldsymbol{T}, C_{2}\right)} \omega\left(C_{2}, C_{2}\right)=-1
$$

Note that $\omega(g, h)= \pm 1$ as elements of 2nd group cohomology $\mathcal{H}^{2}\left(G_{s}, \mathbb{Z}_{2}\right)$, and therefore criterion 67) can be rewritten as follows:

$$
\begin{gathered}
\text { if } n=\text { odd }: \quad \frac{\omega\left(C_{2 n}, \boldsymbol{T}\right)}{\omega\left(\boldsymbol{T}, C_{2 n}\right)}=-\omega_{C_{2 n}} \Leftrightarrow \\
\hat{U}_{C_{2 n}} \boldsymbol{T} \hat{U}_{C_{2 n}}^{-1} \boldsymbol{T}^{-1}=(-1)^{\hat{F}} \cdot\left(\hat{U}_{C_{c n}}\right)^{2 n} .
\end{gathered}
$$

and

$$
\begin{gathered}
\text { if } n=\text { even : } \quad \omega_{C_{2 n}}=-1 \Leftrightarrow \\
\left(\hat{U}_{C_{c n}}\right)^{2 n}=(-1)^{\hat{F}} .
\end{gathered}
$$

In summary, in the presence of a 2-fold rotational symmetry $C_{2}$ centered at a half-integer spin, the 3rd criterion dictates that spinon symmetry fractionalization (67) is incompatible with any gapped symmetric $Z_{2}$ spin liquid. It also establishes the stability of gapless $Z_{2}$ spin liquids protected by such a $C_{2}$ rotational symmetry.

Now that a $2 n$-fold crystalline rotation $C_{2 n}$ necessarily leads to a 2 -fold rotation as shown in $(66)$, our 3rd criterion (67) further leads to two criteria (71)- $(72$ ) for stable gapless $Z_{2}$ spin liquids, protected by a $2 n$-fold rotation $C_{2 n}$ centered at a half-integer spin. Specifically criterion (71) applies to $n=$ odd case, while criterion 72 applies to $n=$ even case.

In the following we apply the above three criteria (38), (58) and (67) to a few examples. These are $Z_{2}$ spin liquids of spin-1/2 systems on square, triangular and kagome lattices with $S U(2)$ spin rotation (it contains $U(1)_{\mathbf{S}^{z}}$ spin rotations as a subgroup), time reversal and space group symmetries. Among all classified symmetric $Z_{2}$ spin liquids in the Abrikosov-fermion representation, where $2 S=1$ and partons $\left\{\phi_{\mathbf{r}, \alpha} \equiv f_{\mathbf{r}, \alpha}\right\}$ are fermions, we will show that a large part of them are gapless $Z_{2}$ spin liquids protected by certain crystalline symmetries. All symmetric gapped $Z_{2}$ spin liquids in the Abrikosov-fermion representation have their counterparts in the Schwingerboson representation, where $2 S=1$ and $\left\{\phi_{\mathbf{r}, \alpha} \equiv b_{\mathbf{r}, \alpha}\right\}$ are hard-core bosons.

\section{GAPPED AND GAPLESS $Z_{2}$ SPIN LIQUIDS ON THE SQUARE LATTICE}

The space group of square lattice is $P 4 \mathrm{gm}$, generated by two translations $T_{1,2}$, mirror reflection $\boldsymbol{\sigma}$ and 


\begin{tabular}{|c|c|c|c|}
\hline Algebraic Identity & $\begin{array}{l}\text { SB } b_{\alpha} \text { in } \\
\text { Ref. [1] }\end{array}$ & $\begin{array}{l}\text { AF } f_{\alpha} \text { in } \\
\text { Ref. } 45\end{array}$ & $\begin{array}{l}\text { fractionalization } \\
\text { class of spinons }\end{array}$ \\
\hline$T_{2}^{-1} T_{1}^{-1} T_{2} T_{1}=\boldsymbol{e}$ & $(-1)^{p_{1}}$ & $\eta_{12}$ & $\omega_{T_{1} T_{2}}$ \\
\hline $\boldsymbol{\sigma}^{-1} T_{1} \boldsymbol{\sigma} T_{2}^{-1}=\boldsymbol{e}$ & 1 & 1 & 1 \\
\hline $\boldsymbol{\sigma}^{-1} T_{2} \boldsymbol{\sigma} T_{1}^{-1}=\boldsymbol{e}$ & 1 & 1 & 1 \\
\hline$C_{6}{ }^{-1} T_{1} C_{6} T_{2}=\boldsymbol{e}$ & 1 & 1 & 1 \\
\hline$C_{6}{ }^{-1} T_{2} C_{6} T_{2}^{-1} T_{1}^{-1}=\boldsymbol{e}$ & 1 & 1 & 1 \\
\hline $\boldsymbol{\sigma}^{2}=\boldsymbol{e}$ & $(-1)^{p_{2}}$ & $\eta_{\boldsymbol{\sigma}}$ & $\omega_{\boldsymbol{\sigma}}$ \\
\hline$R^{2}=\left(C_{6} \boldsymbol{\sigma}\right)^{2}=\boldsymbol{e}$ & $(-1)^{p_{2}+p_{3}}$ & $\eta_{\boldsymbol{\sigma} C_{6}}$ & $\omega_{R}$ \\
\hline$\left(C_{6}\right)^{6}=\boldsymbol{e}$ & $(-1)^{p_{3}}$ & $\eta_{C_{6}}$ & $\omega_{C_{6}}$ \\
\hline$T_{1}^{-1} \boldsymbol{T}^{-1} T_{1} \boldsymbol{T}=\boldsymbol{e}$ & 1 & 1 & 1 \\
\hline$T_{2}^{-1} \boldsymbol{T}^{-1} T_{2} \boldsymbol{T}=\boldsymbol{e}$ & 1 & 1 & 1 \\
\hline $\boldsymbol{\sigma}^{-1} \boldsymbol{T}^{-1} \boldsymbol{\sigma} \boldsymbol{T}=\boldsymbol{e}$ & $(-1)^{p_{2}}$ & $\eta_{\boldsymbol{\sigma} \boldsymbol{T}}$ & $\omega_{\boldsymbol{\sigma} \boldsymbol{T}}$ \\
\hline$R^{-1} \boldsymbol{T}^{-1} R \boldsymbol{T}=\boldsymbol{e}$ & $(-1)^{p_{2}+p_{3}}$ & $\eta_{C_{6} \boldsymbol{T}} \eta_{\boldsymbol{\sigma} \boldsymbol{T}}$ & $\omega_{R \boldsymbol{T}}$ \\
\hline $\boldsymbol{T}^{2}=\boldsymbol{e}$ & -1 & -1 & -1 \\
\hline
\end{tabular}

Table II: Triangular lattice: symmetry fractionalization class $\left(\mathbb{Z}_{2}\right)^{6} \subset \mathcal{H}^{2}\left(P 6 m m \times Z_{2}^{T}, \mathbb{Z}_{2}\right)$ of spinons in half-integer-spin $Z_{2}$ spin liquids, and their realizations in $S=\frac{1}{2}$ Schwingerboson (SB) and Abrikosov-fermion (AF) representations, following the convention of Ref. 3145. The fractionalization classes from 2 nd group cohomology ${ }^{37}$ are labeled by six $Z_{2^{-}}$ valued integers $\omega= \pm 1$. We show that a $Z_{2}$ spin liquid is gapless if violating any of the following conditions: $\omega_{\sigma}=\omega_{\sigma T}$, $\omega_{R}=\omega_{R T}$ and $\omega_{C_{6}}=\omega_{\sigma} \omega_{R}$. This leads to only $2^{3}=8$ distinct gapped $Z_{2}$ spin liquids, all realized in both $\mathrm{SB}^{30 / 31}$ and AF representations $\frac{45}{4}$. There are 12 symmetry protected gapless $Z_{2}$ spin liquids in the AF representation.

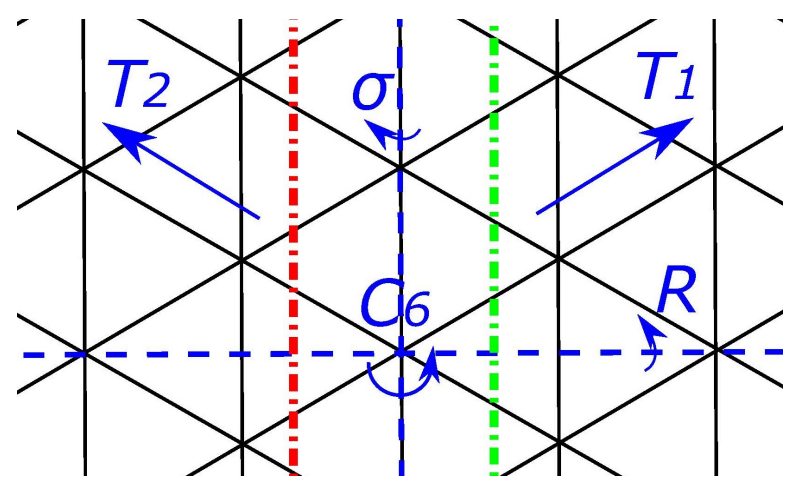

Figure 2: (color online) Space group $(P 6 \mathrm{~mm})$ symmetries of triangular lattice, generated by two translations $T_{1,2}$, mirror reflection $\sigma$ and site-centered 6-fold rotation $C_{6}$. Another mirror reflection is introduced as $R=\left(C_{6}\right)^{3} \sigma$. The two mirror planes $(\boldsymbol{\sigma}$ and $R$ ) intersect at a lattice site. Red and green dotted dash lines denote two entanglement cuts of the lattice, related by mirror $\boldsymbol{\sigma}$ or inversion $I=\left(C_{6}\right)^{3}$.

4-fold rotation $C_{4}$ as shown in FIG. 1. The symmetry implementations on bosonic/fermionic spinons of symmetric $Z_{2}$ spin liquids on the square lattice are summarized in TABLE [ The last column of the table lists the algebraic symmetry fractionalization classes $\left(\mathbb{Z}_{2}\right)^{9} \subset$ $\mathcal{H}^{2}\left(P 4 g m \times Z_{2}^{\boldsymbol{T}}, \mathbb{Z}_{2}\right)$ of spinons, as computed in Ref. 10 .
Since in a half-integer spin system, spinons carry halfinteger spins and are Kramers doublets of time reversal symmetry satisfying $\hat{\boldsymbol{T}}^{2}=(-1)^{\hat{F}}$, each spinon symmetry fractionalization class is labeled by $9 Z_{2}$-valued integers $\{\omega= \pm 1\}^{10}$.

In spin- $1 / 2$ case $(2 S=1)$, Schwinger-boson $(\mathrm{SB})$ and Abrikosov-fermion (AF) representations provide two concrete constructions for symmetric $Z_{2}$ spin liquids associated with the $2^{9}=512$ symmetry fractionalization classes. In the SB representation ${ }^{33}$, the partons $\left\{\phi_{\mathbf{r}, \alpha} \equiv\right.$ $\left.b_{\mathbf{r}, \alpha}\right\}$ are hard-core bosons, while the partons $\left\{\phi_{\mathbf{r}, \alpha} \equiv\right.$ $\left.f_{\mathbf{r}, \alpha}\right\}$ become fermions in the AF representation 34 . A detailed classification ${ }^{32}$ within the SB construction leads to $2^{6}=32$ different states labeled by $6 Z_{2}$-valued integers $\left\{p_{i}=0,1 \mid 1 i=1,2,3,4,7,8\right\}$; while in $\mathrm{AF}$ construction one can write down $176=11 \times 2^{4}$ different $Z_{2}$ spin liquid states $\frac{19}{19}$. In these $\mathrm{SB}$ states, the gaugeinvariant phase factors are given by $6 Z_{2}$-valued phases $\left\{(-1)^{p_{i}}= \pm 1\right\}$, while in AF states they are given by 9 $Z_{2}$-valued phases $\{\eta= \pm 1\}$, as summarized in TABLE[. For instance, the gauge-invariant phase factor $\omega_{T_{1} T_{2}}$ associated with algebraic identity $T_{2}^{-1} T_{1}^{-1} T_{2} T_{1}=e$ is equal to $(-1)^{p_{1}}$ when acting on Schwinger bosons, or $\eta_{x y}= \pm 1$ when acting on Abrikosov fermions. These symmetry implementations on bosonic/fermionic spinons $b_{\alpha} / f_{\alpha}$ in the $\mathrm{SB} / \mathrm{AF}$ representation, as well as their correspondence to the algebraic spinon symmetry fractionalization class are all listed in TABLE I.

Based on our previous analysis, in the general parton construction (1) of half-integer-spin systems, the spinon symmetry fractionalization class satisfying criteria (38), (58), (67) and (71)-(72) will necessarily lead to gapless $Z_{2}$ spin liquids. On the square lattice, there are two perpendicular mirror planes $\boldsymbol{\sigma}$ and $R_{x}=C_{4} \boldsymbol{\sigma}\left(C_{4}\right)^{-1}$ in FIG. 1. that intersects at one lattice site with a spin- $\frac{1}{2}$. Since the two mirror planes are related by a 90 degree rotation $C_{4}$, they share the same gauge-invariant phase factor of spinon symmetry fractionalization

$$
\omega(\boldsymbol{\sigma}, \boldsymbol{\sigma})=\omega\left(R_{x}, R_{x}\right) \equiv \omega_{\boldsymbol{\sigma}} .
$$

The combination of the two mirror is nothing but the 2-fold rotation (or inversion) around a lattice site:

$$
C_{2}=\left(C_{4}\right)^{2}=R_{x} \sigma .
$$

and we have

$$
\omega\left(C_{2}, C_{2}\right)=\omega\left(C_{4}, C_{4}\right) \omega\left(C_{4}, C_{2}\right) \omega\left(C_{4},\left(C_{4}\right)^{3}\right) \equiv \omega_{C_{4}}(75)
$$

Therefore the 1st gapless criterion (38) can be written as

$$
\frac{\omega\left(\boldsymbol{\sigma}, R_{x}\right)}{\omega\left(R_{x}, \boldsymbol{\sigma}\right)}=\frac{\omega\left(C_{2}, C_{2}\right)}{\omega(\boldsymbol{\sigma}, \boldsymbol{\sigma}) \omega\left(R_{x}, R_{x}\right)}=\omega_{C_{4}}=-1 .
$$

on square lattice.

For 2nd gapless criterion (58), its application to mirror plane $\boldsymbol{\sigma}$ leads to

$$
\omega_{\sigma} \omega_{\sigma T}=-1
$$


Meanwhile, applying the same criterion 58 to another mirror plane $R_{x y}=C_{4} \sigma$ leads to

$$
\omega_{R} \omega_{R \boldsymbol{T}}=-1 .
$$

Finally for 4-fold rotational symmetry $C_{4}$, applying the 3rd gapless criterion $(72)$ also leads to condition (76).

To summarize, if any of the above 3 conditions (76)(78) are satisfied for the spinon fractionalization class, we will end up with a symmetry protected gapless $Z_{2}$ spin liquid robust against any symmetry-preserving perturbations. As a result, any gapped symmetric $Z_{2}$ spin liquid on square lattice must obey the following rules of spinon symmetry fractionalization:

$$
\omega_{C_{4}}=\omega_{\boldsymbol{\sigma}} \omega_{\boldsymbol{\sigma} \boldsymbol{T}}=\omega_{R} \omega_{R \boldsymbol{T}}=1 .
$$

This "gap condition" applies to both bosonic and fermionic spinons.

From the 2nd column of TABLEI, it's straightforward to see that the above condition $(79)$ is automatically satisfied for all Schwinger boson mean-field states of $Z_{2}$ spin liquids on square lattice, since

$$
\begin{gathered}
\omega_{C_{4}}^{b}=1, \quad \omega_{\boldsymbol{\sigma}}^{b}=\omega_{\boldsymbol{\sigma} \boldsymbol{T}}^{b}=(-1)^{p_{4}}, \\
\omega_{R}^{b}=\omega_{R \boldsymbol{T}}^{b}=(-1)^{p_{4}+p_{7}} .
\end{gathered}
$$

for the symmetry fractionalization class of bosonic spinons $\left\{b_{\mathbf{r}, \alpha}\right\}$ in the Schwinger-boson (SB) representation. This means all 64 Schwinger boson mean-field states are generically gapped $Z_{2}$ spin liquids ${ }^{32}$.

On the other hand, in the Abrikosov-fermion construction $^{19}$ (3rd column of TABLE I) of symmetric $Z_{2}$ spin liquids on square lattice, the "gap condition" (79) dictates that a gapped $Z_{2}$ spin liquid state must satisfy

$$
\eta_{C_{4}}=\eta_{\sigma} \eta_{\sigma T}=\eta_{\sigma C_{4}} \eta_{C_{4} T}=1 .
$$

since the symmetry fractionalization class of fermionic spinons $\left\{f_{\mathbf{r}, \alpha}\right\}$ are given by

$$
\begin{gathered}
\omega_{C_{4}}^{f}=\eta_{C_{4}}, \quad \omega_{\sigma}^{f}=\eta_{\boldsymbol{\sigma}}, \quad \omega_{\boldsymbol{\sigma} \boldsymbol{T}}^{f}=\eta_{\boldsymbol{\sigma} \boldsymbol{T}}, \\
\omega_{R}^{f}=\eta_{\boldsymbol{\sigma}} \eta_{\boldsymbol{\sigma} C_{4}}, \quad \omega_{R \boldsymbol{T}}^{f}=\eta_{C_{4} \boldsymbol{T}} \eta_{\boldsymbol{\sigma} \boldsymbol{T}} .
\end{gathered}
$$

in the Abrikosov-fermion (AF) representation $\frac{19}{19}$. Among all AF mean-field states, again only $2^{6}=64$ states satisfy the above gap condition and lead to gapped symmetric $Z_{2}$ spin liquids.

One natural question is: what is the relation between the $64 Z_{2}$ spin liquids in $\mathrm{SB}$ representation and the 4 states in AF representation? In fact, it is not coincidental to have 64 gapped symmetric $Z_{2}$ spin liquids in both $\mathrm{SB}$ and $\mathrm{AF}$ representations: there is a one-to-one correspondence between the $64 \mathrm{SB}$ states and the $64 \mathrm{AF}$ states. A gapped $Z_{2}$ spin liquid is topologically ordered 39 and hosts 3 types of gapped anyon excitations: bosonic spinon $b$, fermionic spinon $f$ and bosonic vison $v$ obeying the following fusion rule

$$
f=b \times v
$$

Consequently, the phase factors $\left\{\omega^{a}(g, h) \mid g, h \in G_{s} ; a=\right.$ $b, f, v\}$ characterizing the symmetry fractionalization class of anyons must satisfy the following relation:

$$
\omega^{f}(g, h)=\lambda(g, h) \cdot \omega^{v}(g, h) \cdot \omega^{b}(g, h)
$$

where $\lambda(g, h)= \pm 1$ are extra twisting factors $[10[46] 48$ for symmetry operations $g, h \in G_{s}$. As shown in Ref. 47-49, for any gapped symmetric $Z_{2}$ spin liquid on the square lattice, the vison fractionalization class $\left\{\omega^{v}(g, h)\right\}$ can be uniquely determined. Therefore relation (84) establishes the one-to-one correspondence between bosonic spinon fractionalization class $\left\{\omega^{b}(g, h)\right\}$ and the fermionic spinon one $\left\{\omega^{f}(g, h)\right\}$. In particular, the bosonic partons in SB representation are the bosonic spinons $b$, while fermionic partons in AF representation are the fermionic spinons $f$. Therefore we can establish a duality between the gapped $Z_{2}$ spin liquids in SB representation and those in AF representation. On the square lattice as shown in Ref. 32, this duality map is given by the following identities:

$$
\begin{gathered}
\eta_{x y}=(-1)^{p_{1}+1}, \quad \eta_{x p x}=(-1)^{p_{3}}, \quad \eta_{x p y}=(-1)^{p_{2}+1} \\
\eta_{\boldsymbol{\sigma}}=\eta_{\boldsymbol{\sigma} \boldsymbol{T}}=(-1)^{p_{4}+1}, \quad \eta_{C_{4} \boldsymbol{T}}=\eta_{\boldsymbol{\sigma} C_{4}}=(-1)^{p_{7}} \\
\eta_{t}=(-1)^{p_{8}}, \quad \eta_{C_{4}}=1
\end{gathered}
$$

All $64=4 \times 2^{4}$ gapped $Z_{2}$ spin liquids can be realized by mean-field Ansatz in both SB and AF representations. More concretely in the AF representation, the fermionic partons in all $64=2^{4} \times 4$ gapped $Z_{2}$ spin liquids have the following space group symmetry implementations

$$
\begin{gathered}
\eta_{x y}, \eta_{x p x}, \eta_{x p y}, \eta_{t}= \pm 1 \\
\left(g_{P_{x y}}, g_{P_{x}}, g_{P_{y}}, g_{\boldsymbol{T}}\right)=\left(\tau^{0}, \tau^{0}, \tau^{0}, \mathrm{i} \tau^{3}\right), \\
\left(g_{P_{x y}}, g_{P_{x}}, g_{P_{y}}, g_{\boldsymbol{T}}\right)=\left(\tau^{0}, \mathrm{i} \tau^{1}, \mathrm{i} \tau^{1}, \mathrm{i} \tau^{3}\right), \\
\left(g_{P_{x y}}, g_{P_{x}}, g_{P_{y}}, g_{\boldsymbol{T}}\right)=\left(\mathrm{i} \tau^{1}, \tau^{0}, \tau^{0}, \mathrm{i} \tau^{3}\right), \\
\left(g_{P_{x y}}, g_{P_{x}}, g_{P_{y}}, g_{\boldsymbol{T}}\right)=\left(\mathrm{i} \tau^{1}, \mathrm{i} \tau^{1}, \mathrm{i} \tau^{1}, \mathrm{i} \tau^{3}\right) .
\end{gathered}
$$

in the notation of Ref. 19 .

While all SB mean-field states are gapped $Z_{2}$ spin liquids, many states in the $\mathrm{AF}$ representation $\sqrt{1920}$ correspond to symmetry-protected gapless $Z_{2}$ spin liquids. In particular, in total there are $160=10 \times 2^{4} \mathrm{AF}$ meanfield states where fermionic spinons $\left\{f_{\mathbf{r}, \alpha}\right\}$ are Kramers doublets of time reversal symmetry with $\boldsymbol{T}^{2}=(-1)^{\hat{F}}$. Besides the 64 gapped $Z_{2}$ spin liquids, the remaining $6 \times 2^{4}=96 \mathrm{AF}$ states with Kramers-doublet spinons all satisfy at least one of the 3 gapless conditions $(76)$ (78). Therefore as we discussed, these 96 states in Ref. 19 all realize symmetry-protected gapless $Z_{2}$ spin liquids, which are robust against any symmetry-preserving perturbations. 


\begin{tabular}{|c|c|c|c|}
\hline Algebraic Identity & $\begin{array}{l}\text { SB } b_{\alpha} \text { in } \\
\text { Ref. 31 }\end{array}$ & $\begin{array}{l}\text { AF } f_{\alpha} \text { in } \\
\text { Ref. [50 }\end{array}$ & $\begin{array}{c}\text { fractionalization } \\
\text { class of spinons }\end{array}$ \\
\hline$T_{2}^{-1} T_{1}^{-1} T_{2} T_{1}$ & $(-1)^{p_{1}}$ & $\eta_{12}$ & $\omega_{T_{1} T_{2}}$ \\
\hline$T_{1}^{-1} C_{6}{ }^{-1} T_{2} C_{6}$ & 1 & 1 & 1 \\
\hline$T_{1}^{-1} T_{2} C_{6}{ }^{-1} T_{1} C_{6}$ & 1 & 1 & 1 \\
\hline$T_{1}^{-1} R_{y}^{-1} T_{1} R_{y}$ & $(-1)^{p_{1}}$ & $\eta_{12}$ & $\omega_{T_{1} T_{2}}$ \\
\hline$T_{1}^{-1} T_{2} R_{y}^{-1} T_{2} R_{y}$ & $(-1)^{p_{1}}$ & $\eta_{12}$ & $\omega_{T_{1} T_{2}}$ \\
\hline$R_{x}^{2}=\left(C_{6} R_{y}\right)^{2}$ & $(-1)^{p_{2}+p_{3}}$ & $\eta_{\boldsymbol{\sigma}}$ & $\omega_{R_{x}}$ \\
\hline$\left(R_{y}\right)^{2}$ & $(-1)^{p_{2}}$ & $\eta_{\boldsymbol{\sigma}} \eta_{\boldsymbol{\sigma} C_{6}}$ & $\omega_{R_{y}}$ \\
\hline$\left(C_{6}\right)^{6}=I^{2}$ & $(-1)^{p_{1}+p_{3}}$ & $\eta_{C_{6}}$ & $\omega_{C_{6}}$ \\
\hline$T_{1}^{-1} \boldsymbol{T}^{-1} T_{1} \boldsymbol{T}$ & 1 & 1 & 1 \\
\hline$T_{2}^{-1} \boldsymbol{T}^{-1} T_{2} \boldsymbol{T}$ & 1 & 1 & 1 \\
\hline$R_{y}^{-1} \boldsymbol{T}^{-1} R_{y} \boldsymbol{T}$ & $(-1)^{p_{2}}$ & $\eta_{\boldsymbol{\sigma} \boldsymbol{T}} \eta_{C_{6} \boldsymbol{T}}$ & $\omega_{R_{y} \boldsymbol{T}}$ \\
\hline$R_{x}^{-1} \boldsymbol{T}^{-1} R_{x} \boldsymbol{T}$ & $(-1)^{p_{2}+p_{3}}$ & $\eta_{\boldsymbol{\sigma} \boldsymbol{T}}$ & $\omega_{R_{x} \boldsymbol{T}}$ \\
\hline $\boldsymbol{T}^{2}$ & -1 & -1 & -1 \\
\hline
\end{tabular}

Table III: Kagome lattice: symmetry fractionalization class $\left(\mathbb{Z}_{2}\right)^{6} \subset \mathcal{H}^{2}\left(P 6 m m \times Z_{2}^{T}, \mathbb{Z}_{2}\right)$ of spinons in half-integer-spin $Z_{2}$ spin liquids, and their realizations in $S=1 / 2$ Schwingerboson (SB) and Abrikosov-fermion (AF) representations, following the convention of Ref. 31150 The fractionalization classes from 2nd group cohomology ${ }^{46}$ are labeled by six $Z_{2^{-}}$ valued integers $\omega= \pm 1$. We show that a $Z_{2}$ spin liquid is gapless if violating any of the following conditions: $\omega_{R_{x}}=\omega_{R_{x} T}$, $\omega_{R_{y}}=\omega_{R_{y} T}$ and $\omega_{T_{1} T_{2}} \omega_{C_{6}}=\omega_{R_{x}} \omega_{R_{y}}$. This leads to only $2^{3}=8$ distinct gapped $Z_{2}$ spin liquids, all realized in both $\mathrm{SB}^{30131}$ and $\mathrm{AF}^{50}$ representations. There are 12 symmetry protected gapless $Z_{2}$ spin liquids in the $\mathrm{AF}$ construction.

\section{GAPPED AND GAPLESS $Z_{2}$ SPIN LIQUIDS ON THE TRIANGULAR LATTICE}

As shown in FIG. 2, the space group $P 6 \mathrm{~mm}$ of triangular lattice is generated by translations $T_{1,2}$, mirror reflection $\sigma$ and 6 -fold site-centered rotation $C_{6}$. The symmetry fractionalization class of spinons is classified ${ }^{37}$ as $\left(\mathbb{Z}_{2}\right)^{6} \subset \mathcal{H}^{2}\left(P 6 m m \times Z_{2}^{T}, \mathbb{Z}_{2}\right)$, labeled by $6 Z_{2}$-valued coefficients $\omega= \pm 1$ in the last column of TABLE [I] Again only a part of these $Z_{2}$ spin liquids are realized in spin- $\frac{1}{2}(2 S=1)$ parton constructions, leading to $2^{3}=8$ Schwinger-boson (SB) states ${ }^{31}$ and 20 Abrikosov-fermion (AF) state $37 / 45$. In fermionic parton construction (1) of spin-3/2 $(2 S=3)$ systems, Ref. 37/ showed that all $2^{6}=64$ symmetry fractionalization classes of fermionic spinons can be realized.

Now we utilize the gapless criteria (38), (58) and (71) to identify gapless $Z_{2}$ spin liquids among all states. First, applying criterion (38) to two perpendicular mirror planes $\boldsymbol{\sigma}$ and $R \equiv\left(C_{6}\right)^{3} \boldsymbol{\sigma}$ in FIG. 2 leads to one gapless condition:

$$
\frac{\omega(\boldsymbol{\sigma}, R)}{\omega(R, \boldsymbol{\sigma})}=\frac{\omega\left(C_{2}, C_{2}\right)}{\omega(\boldsymbol{\sigma}, \boldsymbol{\sigma}) \cdot \omega(R, R)}=\omega_{\boldsymbol{\sigma}} \omega_{R} \omega_{C_{6}}=-1 .
$$

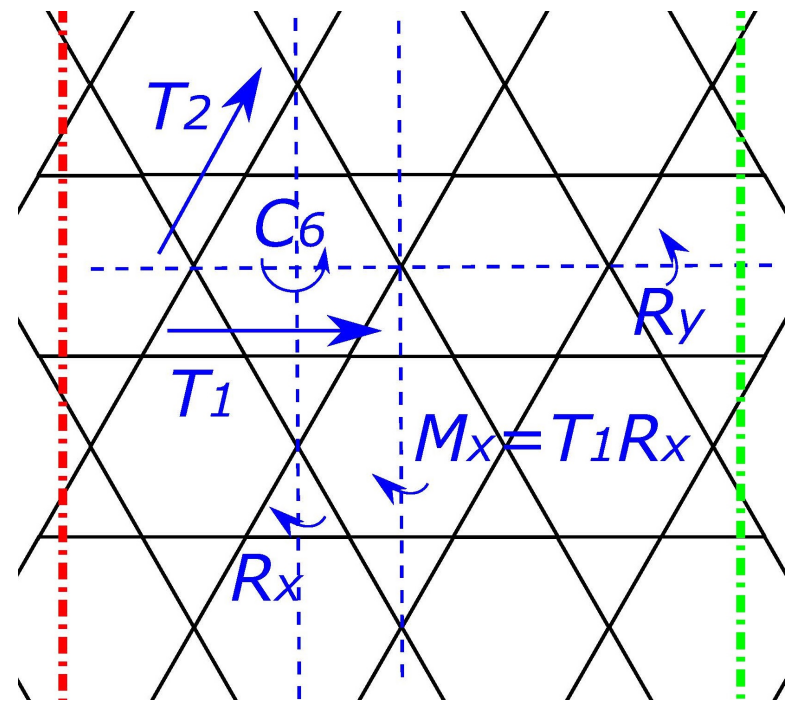

Figure 3: (color online) Space group $(P 6 \mathrm{~mm})$ symmetries of kagome lattice, generated by two translations $T_{1,2}$, mirror reflection $R_{y}$ and hexagon-centered 6 -fold rotation $C_{6}$. Two extra mirror symmetries are introduced as $R_{x}=\left(C_{6}\right)^{3} \boldsymbol{\sigma}$ and $M_{x}=T_{1} R_{x}$. Two mirror planes, $R_{y}$ and $M_{x}$, intersect at a lattice site. Red and green dotted dash lines denote two entanglement cuts of the lattice, related by mirror $M_{x}$ or inversion $M_{x} R_{y}$.

where we defined 2-fold rotation (or inversion) around a site

$$
C_{2} \equiv\left(C_{6}\right)^{3}=\sigma \cdot R
$$

Next, applying criterion (58) to mirror plane $\boldsymbol{\sigma}$ and $R$ leads to two gapless conditions on the fractionalization class in TABLE[I]

$$
\begin{aligned}
& \omega_{\boldsymbol{\sigma}} \omega_{\boldsymbol{\sigma} \boldsymbol{T}}=-1 \\
& \omega_{R} \omega_{R \boldsymbol{T}}=-1
\end{aligned}
$$

Finally, criterion $(71)$ for site-centered $C_{6}$ rotation also leads to another gapless condition

$$
\frac{\omega\left(C_{6}, \boldsymbol{T}\right)}{\omega\left(\boldsymbol{T}, C_{6}\right)}=\omega_{\boldsymbol{\sigma} \boldsymbol{T}} \cdot \omega_{R \boldsymbol{T}}=-\omega_{C_{6}} .
$$

This condition, however, is not independent of the above gapless conditions $866-(88)$. Any spinon symmetry fractionalization class $\{\omega(g, h)\}$ that satisfies at least one of conditions 866-(88) will inevitably lead to a gapless spectrum in the $Z_{2}$ spin liquid.

This means any gapped symmetric $Z_{2}$ spin liquid on the triangular lattice must satisfy following "gap condition"

$$
\omega_{\boldsymbol{\sigma}} \omega_{R} \omega_{C_{6}}=\omega_{\boldsymbol{\sigma}} \omega_{\boldsymbol{\sigma} \boldsymbol{T}}=\omega_{R} \omega_{R \boldsymbol{T}}=1 .
$$

on the fractionalizaton class of either fermionic or bosonic spinons.

For all 8 Schwinger-boson states ${ }^{31}$ listed in 2 nd column of TABLE II] it's straightforward to see that "gap 
conditions" 89 are automatically fulfilled, since the fractionalization class $\left\{\omega^{b}(g, h)\right\}$ of bosonic spinons $\left\{b_{\mathbf{r}, \alpha}\right\}$ is given by

$$
\begin{gathered}
\omega_{\boldsymbol{\sigma}}^{b}=\omega_{\boldsymbol{\sigma} \boldsymbol{T}}^{b}=(-1)^{p_{2}}, \quad \omega_{R}^{b}=\omega_{R \boldsymbol{T}}^{b}=(-1)^{p_{2}+p_{3}}, \\
\omega_{C_{6}}^{b}=(-1)^{p_{3}} .
\end{gathered}
$$

in the SB representation.

On the other hand, for the $20 \mathrm{AF}$ states ${ }^{45}$ listed in the 3rd column of TABLE II the gap condition 89 leads to

$$
\eta_{\boldsymbol{\sigma}}=\eta_{\boldsymbol{\sigma} \boldsymbol{T}}=\eta_{\boldsymbol{\sigma} C_{6}} \eta_{C_{6}}, \quad \eta_{C_{6}}=\eta_{C_{6} \boldsymbol{T}}
$$

The symmetry fractionalization class $\left\{\omega^{f}(g, h)\right\}$ of fermionic spinons $\left\{f_{\mathbf{r}, \alpha}\right\}$ is given by

$$
\begin{gathered}
\omega_{\boldsymbol{\sigma}}^{f}=\eta_{\boldsymbol{\sigma}}, \quad \omega_{\boldsymbol{\sigma} \boldsymbol{T}}^{f}=\eta_{\boldsymbol{\sigma} \boldsymbol{T}}, \quad \omega_{C_{6}}=\eta_{C_{6}}, \\
\omega_{R}^{f}=\eta_{\boldsymbol{\sigma} C_{6}}, \quad \omega_{R \boldsymbol{T}}^{f}=\eta_{C_{6} \boldsymbol{T}} \eta_{\boldsymbol{\sigma} \boldsymbol{T}} .
\end{gathered}
$$

in the AF representation. Only 8 states among all 20 satisfy the above gap conditions $(91)$, and lead to gapped symmetric $Z_{2}$ spin liquids on triangular lattice.

Similar to the square lattice case, these 8 AF states have a one-to-one correspondence with the $8 \mathrm{SB}$ states on triangular lattice. As shown in Ref. 45, the duality between SB and AF states are established in terms of their bosonic/fermionic fractionalization classes:

$$
\begin{gathered}
\eta_{12}=(-1)^{p_{1}+1}, \quad \eta_{C_{6}}=\eta_{C_{6} \boldsymbol{T}}=(-1)^{p_{3}}, \\
\eta_{\boldsymbol{\sigma}}=\eta_{\boldsymbol{\sigma} \boldsymbol{T}}=\eta_{C_{6}} \eta_{\boldsymbol{\sigma} C_{6}}=(-1)^{p_{2}+1} .
\end{gathered}
$$

Therefore they both describe the 8 distinct gapped symmetric $Z_{2}$ spin liquids on the triangular lattice.

In addition to the 8 gapped $Z_{2}$ spin liquids, there are 12 other mean-field states in the AF representation of a spin- $\frac{1}{2}$ system on triangular lattice $\sqrt{37 / 45}$. They all satisfy at least one of gapless conditions (86)-(88). As a result, there are 12 symmetry protected gapless $Z_{2}$ spin liquids realized in the AF representation, which are robust against any symmetry-preserving perturbations on the triangular lattice.

\section{GAPPED AND GAPLESS $Z_{2}$ SPIN LIQUIDS ON THE KAGOME LATTICE}

Kagome lattice share the same space group $P 6 \mathrm{~mm}$ with triangular lattice, generated by translations $T_{1,2}$, mirror reflection $R_{y}$ and hexagon-centered rotation $C_{6}$, as shown in FIG. 3 Therefore spinon fractionalization class on kagome lattice is also classified $\frac{\sqrt{46}}{}$ by $\left(\mathbb{Z}_{2}\right)^{6} \subset$ $\mathcal{H}^{2}\left(P 6 m m \times Z_{2}^{\boldsymbol{T}}, \mathbb{Z}_{2}\right)$, characterized by $6 Z_{2}$-valued coefficients $\omega= \pm 1$ in the last column of TABLE [II] In the parton constructions of a spin- $\frac{1}{2}(2 S=1)$ system on the kagome lattice, Schwinger-boson (SB) representation leads to $2^{3}=8$ different $Z_{2}$ spin liquid states ${ }^{31}$, while Abrikosov-fermion (AF) representation gives rise to 20 different states 50 . Below we utilize gapless criteria (38), (58) and (71) to identify gapped and gapless $Z_{2}$ spin liquids among them.

According to the 1st criterion (38), two perpendicular mirror planes $M_{x}=T_{1} R_{x}$ and $R_{y}$ in FIG. 3 intersect at a lattice site, leading to the following gapless condition on the spinon fractionalization class

$$
\begin{gathered}
\frac{\omega\left(M_{x}, R_{y}\right)}{\omega\left(R_{y}, M_{x}\right)}=\frac{\omega_{T_{1}, R_{y}}}{\omega_{R_{y}, T_{1}}} \cdot \frac{\omega\left(R_{x}, R_{y}\right)}{\omega\left(R_{y}, R_{x}\right)}= \\
\omega_{T_{1} T_{2}} \omega_{R_{x}} \omega_{R_{y}} \omega_{C_{6}}=-1 .
\end{gathered}
$$

Next, applying 2nd criterion (58) to mirror reflections $R_{x}$ and $R_{y}$ separately leads to two gapless conditions:

$$
\begin{aligned}
& \omega_{R_{x}} \omega_{R_{x} T}=-1 \\
& \omega_{R_{y}} \omega_{R_{y} T}=-1
\end{aligned}
$$

Finally, applying 3rd criterion (71) to site-centered 2-fold rotation or inversion

$$
I \equiv M_{x} R_{y}=T_{1} R_{x} R_{y}=T_{1}\left(C_{6}\right)^{3}
$$

lead to another gapless condition

$$
\begin{aligned}
& \frac{\omega(I, \boldsymbol{T})}{\omega(\boldsymbol{T}, I)}=\omega_{R_{x} \boldsymbol{T}} \omega_{R_{y} \boldsymbol{T}} \\
&=-\omega(I, I)=-\omega_{C_{6}} \omega_{T_{1} T_{2}}
\end{aligned}
$$

Again, it's straightforward to show this condition is not independent of the above 3 gapless conditions (94)-967). Satisfying one or more of these 3 gapless conditions necessarily result in a stable gapless $Z_{2}$ spin liquid.

In order to achieve a gapped symmetric $Z_{2}$ spin liquid on the kagome lattice, the symmetry fractionalization class of both bosonic and fermionic spinons hence must obey the following gap condition

$$
\omega_{T_{1} T_{2}} \omega_{R_{x}} \omega_{R_{y}} \omega_{C_{6}}=\omega_{R_{x}} \omega_{R_{x} \boldsymbol{T}}=\omega_{R_{y}} \omega_{R_{y} \boldsymbol{T}}=1 .
$$

Similar to square and triangular lattices, as shown in 2nd column in TABLE III all 8 spin- $\frac{1}{2}$ Schwinger-boson $Z_{2}$ spin liquid states on kagome lattice ${ }^{31}$ automatically satisfy the above gap conditions 97). In particular, the fractionalization class of bosonic spinons $\left\{b_{\mathbf{r}, \alpha}\right\}$ is given by

$$
\begin{gathered}
\omega_{T_{1} T_{2}}^{b}=(-1)^{p_{1}}, \quad \omega_{R_{y}}^{b}=\omega_{R_{y} \boldsymbol{T}}^{b}=(-1)^{p_{2}}, \\
\omega_{C_{6}}^{b}=(-1)^{p_{1}+p_{3}}, \quad \omega_{R_{x}}^{b}=\omega_{R_{x} T}^{b}=(-1)^{p_{2}+p_{3}} .
\end{gathered}
$$

in the SB representation. All 8 SB states are gaped symmetric $Z_{2}$ spin liquids.

On the other hand, for $\mathrm{AF} Z_{2}$ spin liquid states in 3rd column of TABLEIII, fractionalization class of fermionic spinons $\left\{f_{\mathbf{r}, \alpha}\right\}$ is given by

$$
\begin{gathered}
\omega_{T_{1} T_{2}}^{f}=\eta_{12}, \quad \omega_{R_{x}}^{f}=\eta_{\boldsymbol{\sigma}}, \quad \omega_{R_{x} \boldsymbol{T}}^{f}=\eta_{\boldsymbol{\sigma} \boldsymbol{T}}, \\
\omega_{C_{6}}^{f}=\eta_{C_{6}}, \quad \omega_{R_{y}}^{b}=\eta_{\boldsymbol{\sigma}} \eta_{\boldsymbol{\sigma} C_{6}}, \quad \omega_{R_{y} \boldsymbol{T}}^{f}=\eta_{\boldsymbol{\sigma} \boldsymbol{T}} \eta_{C_{6} \boldsymbol{T}} .
\end{gathered}
$$


in the AF representation. The gap condition (97) hence provides the following constraints on the fractionalization class of fermonic spinons:

$$
\eta_{12} \eta_{C_{6}} \eta_{\boldsymbol{\sigma} C_{6}}=\eta_{\boldsymbol{\sigma}} \eta_{\boldsymbol{\sigma} \boldsymbol{T}}=\eta_{\boldsymbol{\sigma} C_{6}} \eta_{C_{6} \boldsymbol{T}}=1 .
$$

Among all 20 AF states 50 , only 8 fulfill this constraint and lead to gapped symmetric $Z_{2}$ spin liquids.

Analogous to the case of square and triangular lattices, there is a one-to-one correspondence between the $8 \mathrm{SB}$ states and 8 gapped AF states, established by the unique vision fractionalization class in a symmetric $Z_{2}$ spin liquid on the square lattice. As shown in Ref.48, the duality between $8 \mathrm{SB}$ and $8 \mathrm{AF}$ states is given by

$$
\begin{gathered}
\eta_{12}=(-1)^{p_{1}+1}, \quad \eta_{\boldsymbol{\sigma}}=\eta_{\boldsymbol{\sigma T}}=(-1)^{p_{2}+p_{3}+1} \\
\eta_{C_{6} \boldsymbol{T}}=\eta_{\boldsymbol{\sigma} C_{6}}=(-1)^{p_{2}}, \quad \eta_{C_{6}}=(-1)^{p_{1}+p_{3}+1}
\end{gathered}
$$

Hence there are 8 gapped symmetric $Z_{2}$ spin liquids on the kagome lattice, realizable in both $\mathrm{SB}$ and $\mathrm{AF}$ constructions.

In addition to the 8 gapped states, the other 12 states in AF representation all satisfy at least one of gapless conditions (94)-(96). They realize 12 different gapless $Z_{2}$ spin liquids on the kagome lattice, stable against any symmetry-preserving perturbations.

\section{DISCUSSIONS}

In this work we establish a class of gapless topological phases in half-integer spin systems, which hosts symmetry-protected low-energy bulk excitations stable against any symmetric perturbations. These phases require time reversal, certain space group and at least $U(1)$ spin rotational symmetries. The low-energy dynamics of these phases are described by a (gapped) dynamical $Z_{2}$ gauge field coupled to gapless mater fields, halfinteger "spinons" that transform as a Kramers doublet under time reversal symmetry. Therefore these phases are dubbed "gapless $Z_{2}$ spin liquids".

In the framework of parton constructions for halfinteger spin systems, these gapless $Z_{2}$ spin liquids are constructed as spin-conserving pair superfluids of spinons (or partons). We establish three criteria (38), (58), (71)(72) regarding the spatial (and time reversal) symmetry implementations on spinons. These criteria forbid a gapped spinon pair superfluid for arbitrary interactions between spinons. The non-perturbative proof for gapless pair superfluids of interacting spinons is achieved by using properties of Schmidt decomposition of a generic SRE state 25. These criteria naturally lead to nonperturbative necessary conditions for a gapped pair superfluid of interacting (hard-core) spinons.

We apply these gapless criteria to symmetric $Z_{2}$ spin liquids on square, triangular and kagome lattices, and

found that the only gapped $Z_{2}$ spin liquids that can be realized on these lattices are the Schwinger-boson states 30 . There are only $2^{6}=64$ gapped $Z_{2}$ spin liquids on square lattice, and $2^{3}=8$ gapped states on triangular or kagome lattice. All these states can also be realized in the Abrikosov-fermion representation 19|45|50. In addition to these gapped states, there is also a large number of symmetry protected gapless spin-1/2 $Z_{2}$ spin liquids in Abrikosov-fermion construction: 96 on square lattice, 12 on kagome or triangular lattice. They are stable against any symmetry-preserving perturbations.

One thing worth mentioning is that these nonperturbative gapless criteria for a pair superfluid of interacting hard-core bosons/fermions hold quite generally, regardless of the topology of the ground state. In other words, they can be applied to a trivial pair superfluid or a topological pair superfluid with protected edge states ${ }^{6 / 51}$. Once coupled to a dynamical $Z_{2}$ gauge field, a topological pair superfluid can describe other topological orders (such as double semion theory ${ }^{52}$ ) other than $Z_{2}$ spin liquids. One future direction is to apply these criteria to other interacting topological phases.

Note added: Upon completion of this work we became aware of Qi and Cheng's work ${ }^{53}$, which classified all gapped symmetric $Z_{2}$ spin liquids on kagome/triangular lattice in the generic formalism of symmetry fractionalization. Their results agree with ours from parton construction on kagome/triangular lattices.

\section{Acknowledgments}

I thank Ashvin Vishwanath and Mike Zaletel for previous collaborations, as well as Yang Qi and Meng Cheng for sending me their draft. This work is supported by startup funds at Ohio State University.
1 T. Senthil, Annual Review of Condensed Matter Physics, Annu. Rev. Condens. Matter Phys. 6, 299 (2015).

2 C.-K. Chiu, J. C. Y. Teo, A. P. Schnyder, and S. Ryu, Rev. Mod. Phys. 88, 035005 (2016).
3 X.-G. Wen, Rev. Mod. Phys. 89, 041004 (2017)

${ }^{4}$ M. Z. Hasan and C. L. Kane, Rev. Mod. Phys. 82, 3045 (2010)

${ }^{5}$ M. Z. Hasan and J. E. Moore, Annual Review of Condensed 
Matter Physics, Annu. Rev. Condens. Matter Phys. 2, 55 (2011).

${ }^{\circ}$ X.-L. Qi and S.-C. Zhang, Rev. Mod. Phys. 83, 1057 (2011)

7 X. Chen, Z.-C. Gu, Z.-X. Liu, and X.-G. Wen, Phys. Rev. B 87, 155114 (2013)

${ }^{8}$ Y.-M. Lu and A. Vishwanath, Phys. Rev. B 86, 125119 (2012).

${ }^{9}$ Z. Bi, A. Rasmussen, K. Slagle, and C. Xu, Phys. Rev. B 91, 134404 (2015)

io A. M. Essin and M. Hermele, Phys. Rev. B 87, 104406 (2013).

11 A. Mesaros and Y. Ran, Phys. Rev. B 87, 155115 (2013)

12 L.-Y. Hung and X.-G. Wen, Phys. Rev. B 87, 165107 (2013)

is Y.-M. Lu and A. Vishwanath, Phys. Rev. B 93, 155121 (2016).

${ }^{14}$ M. Barkeshli, P. Bonderson, M. Cheng, and Z. Wang, ArXiv e-prints (2014), arXiv:1410.4540 [cond-mat.str-el].

15 J. C. Y. Teo, T. L. Hughes, and E. Fradkin, Annals of Physics 360, 349 (2015).

${ }^{10}$ N. Tarantino, N. H. Lindner, and L. Fidkowski, New Journal of Physics 18, 035006 (2016)

17 A. M. Turner and A. Vishwanath, ArXiv e-prints (2013), arXiv:1301.0330 [cond-mat.str-el] .

18 N. P. Armitage, E. J. Mele, and A. Vishwanath, ArXiv e-prints (2017), arXiv:1705.01111 [cond-mat.str-el].

19 X.-G. Wen, Phys. Rev. B 65, 165113 (2002)

20 X. G. Wen and A. Zee, Phys. Rev. B 66, 235110 (2002)

21 N. Read and S. Sachdev, Phys. Rev. Lett. 66, 1773 (1991)

${ }^{22}$ X. G. Wen, Phys. Rev. B 44, 2664 (1991)

23 S. Matsuura, P.-Y. Chang, A. P. Schnyder, and S. Ryu, New Journal of Physics 15, 065001 (2013).

24 C.-K. Chiu and A. P. Schnyder, Phys. Rev. B 90, 205136 (2014).

${ }^{25}$ F. Pollmann, A. M. Turner, E. Berg, and M. Oshikawa, Phys. Rev. B 81, 064439 (2010).

26 X. Chen, Z.-C. Gu, and X.-G. Wen, Phys. Rev. B 83, 035107 (2011).

${ }^{27}$ H. Watanabe, H. C. Po, A. Vishwanath, and M. Zaletel, Proceedings of the National Academy of Sciences 112, 14551 (2015)

${ }_{28}$ P. Mendels, F. Bert, M. A. de Vries, A. Olariu, A. Harrison, F. Duc, J. C. Trombe, J. S. Lord, A. Amato, and C. Baines, Phys. Rev. Lett. 98, 077204 (2007).

29 M. Fu, T. Imai, T.-H. Han, and Y. S. Lee, Science 350, 655 (2015).

30 S. Sachdev, Phys. Rev. B 45, 12377 (1992).

${ }^{31}$ F. Wang and A. Vishwanath, Phys. Rev. B 74, 174423 (2006)

${ }^{32}$ X. Yang and F. Wang, Phys. Rev. B 94, 035160 (2016).
33 J. Schwinger, "Quantum theory of angular momentum," (Academic Press, New York, 1965) Chap. On angular momentum, p. 229.

34 A. A. Abrikosov, Physics 2, 5 (1965).

${ }^{35}$ C. Xu, F. Wang, Y. Qi, L. Balents, and M. P. A. Fisher, Phys. Rev. Lett. 108, 087204 (2012)

36 F. Wang and C. Xu, ArXiv e-prints (2011), arXiv:1110.4091 [cond-mat.str-el] .

37 W. Zheng, J.-W. Mei, and Y. Qi, ArXiv e-prints (2015), arXiv:1505.05351 [cond-mat.str-el] .

38 I. Affleck, Z. Zou, T. Hsu, and P. W. Anderson, Phys. Rev. B 38, 745 (1988)

39 X.-G. Wen, Quantum Field Theory Of Many-body Systems: From The Origin Of Sound To An Origin Of Light And Electrons (Oxford University Press, New York, 2004).

${ }^{40}$ M. Oshikawa and T. Senthil, Phys. Rev. Lett. 96, 060601 (2006)

${ }^{41}$ M. P. Zaletel and A. Vishwanath, Phys. Rev. Lett. 114, 077201 (2015)

42 M. Cheng, M. Zaletel, M. Barkeshli, A. Vishwanath, and P. Bonderson, Phys. Rev. X 6, 041068 (2016).

43 L. Capriotti, F. Becca, A. Parola, and S. Sorella, Phys. Rev. Lett. 87, 097201 (2001)

${ }^{44}$ W.-J. Hu, F. Becca, A. Parola, and S. Sorella, Phys. Rev. B 88, $060402(2013)$

45 Y.-M. Lu, Phys. Rev. B 93, 165113 (2016).

46 Y. Qi and L. Fu, Phys. Rev. B 91, 100401 (2015).

47 M. P. Zaletel, Y.-M. Lu, and A. Vishwanath, Phys. Rev. B 96, 195164 (2017)

48 Y.-M. Lu, G. Y. Cho, and A. Vishwanath, Phys. Rev. B 96, $205150(2017)$

49 Y. Qi, M. Cheng, and C. Fang, ArXiv e-prints (2015), arXiv:1509.02927 [cond-mat.str-el] .

50 Y.-M. Lu, Y. Ran, and P. A. Lee, Phys. Rev. B 83, 224413 (2011)

ग1 A. P. Schnyder, S. Ryu, A. Furusaki, and A. W. W. Ludwig, Phys. Rev. B 78, 195125 (2008)

52 M. A. Levin and X.-G. Wen, Phys. Rev. B 71, 045110 (2005)

53 Y. Qi and M. Cheng, ArXiv e-prints (2016), arXiv:1606.04544 [cond-mat.str-el] .

54 In comparison, the symmetry implementations on the parton Fock vacuum $|0\rangle$ defined in $(26)$ are quite different: $\hat{U}_{g} \cdot \hat{U}_{h}|0\rangle=[\omega(g, h)]^{2 N S} \hat{U}_{g h}|0\rangle$ for all $g, h \in G_{s}$, with the only exception that $\hat{\boldsymbol{T}}^{2}|0\rangle=|0\rangle$.

55 A crucial difference between an even-rank rotation $C_{2 n}$ and odd-rank rotation $C_{2 n+1}$ is that two-dimensional inversion $\hat{I}=\hat{C}_{2}$ can be generated by even-rank rotation $I=\left(C_{2 n}\right)^{n}$ but not the odd-rank rotation. Therefore our 3rd criterion only applies to even-rank rotation $C_{2 n}$. 